\title{
Confronting the fourth generation two-Higgs-doublet model with the phenomenology of heavy Higgs bosons
}

\author{
Sin Kyu Kang, ${ }^{1}$ Zhuoni Qian, ${ }^{2}$ Jeonghyeon Song, ${ }^{3}$ and Yeo Woong Yoon ${ }^{3}$ \\ ${ }^{1}$ School of Liberal Arts, Seoul National University of Science and Technology, Seoul 01811, Korea \\ ${ }^{2}$ Center for Theoretical Physics of the Universe, Institute for Basic Science (IBS), Daejeon, 34126, Korea \\ ${ }^{3}$ Department of Physics, Konkuk University, Seoul 05029, Korea
}

(Received 16 October 2018; published 27 November 2018)

\begin{abstract}
A sequential fourth generation is known to be excluded because the nondecoupling contribution to $\kappa_{g}$, the Higgs coupling modifier with a gluon pair, is unacceptably large. Recently a new way to save the model was suggested in the type-II two-Higgs-doublet model: If the Yukawa couplings of down-type fermions have the wrong sign, the contributions from $t^{\prime}$ and $b^{\prime}$ to $\kappa_{g}$ are canceled. We study the theoretical and experimental constraints on this model, focusing on the heavy Higgs bosons. We point out two constraining features. First, the exact wrong-sign limit does not allow alignment, which causes the perturbative unitarity for the scalar-scalar scattering to put upper bounds on the heavy Higgs boson masses like $M_{H}, M_{A} \lesssim$ $920 \mathrm{GeV}$ and $M_{H^{ \pm}} \lesssim 620 \mathrm{GeV}$. Second, the Yukawa couplings of the fourth generation fermions to the heavy Higgs bosons are generically large, being proportional to the heavy fermion mass and, for the downtype fermions, to $\tan \beta$ as well. The gluon fusion production of $H$ and $A$ through the fourth generation quark loops becomes significant. We find that the current LHC data on $p p \rightarrow Z Z$ for $H$, along with the theoretical and indirect constraints, exclude the model at leading order.
\end{abstract}

DOI: 10.1103/PhysRevD.98.095025

\section{INTRODUCTION}

Our greatest hope for new physics (NP) beyond the standard model (SM) has not been fulfilled yet since the dedicated searches for new particles at the LHC found no new signals, and various precision data are consistent with the SM predictions. The usual strategy for a NP theory is to hide it in the decoupling limit: Very heavy new particles cannot be observed at the current $13 \mathrm{TeV}$ LHC, nor do they significantly contribute to the precision data, including the Higgs signals. One important exception is a sequential fourth generation model where the new chiral fermions $\left(t^{\prime}\right.$, $b^{\prime}, \nu^{\prime}$ and $\tau^{\prime}$ ) acquire their masses via the same Higgs mechanism. This model is motivated by the question of whether there exist only three fermion generations in the Universe [1-3]. The discovery of the SM-like Higgs boson with a mass of $125 \mathrm{GeV}[4,5]$ excludes this model since the contributions of the heavy fourth generation quarks to the gluon fusion production of the Higgs boson do not decouple but saturate to a constant value which is unacceptably large.

Published by the American Physical Society under the terms of the Creative Commons Attribution 4.0 International license. Further distribution of this work must maintain attribution to the author(s) and the published article's title, journal citation, and DOI. Funded by SCOAP ${ }^{3}$.
Based on the observation that the NP contribution to the Higgs coupling modifier with a gluon pair, $\kappa_{g}$, is proportional to the sum of the Higgs coupling modifiers of $t^{\prime}$ and $b^{\prime}$, i.e., $\left(\kappa_{t^{\prime}}+\kappa_{b^{\prime}}\right)$ when $M_{t^{\prime}}, M_{b^{\prime}} \gg m_{h}$, the authors of Ref. [6] found that the sequential fourth generation model can survive the Higgs precision constraint if the down-type quark Yukawa coupling has opposite sign to that of the up-type quark, i.e., $\kappa_{b^{\prime}}=-\kappa_{t^{\prime}}$. More interesting is that in the exact wrongsign limit where all of the down-type fermions have opposite Higgs coupling to the up-type fermion, the new contributions to $\kappa_{\gamma \gamma}$ and $\kappa_{Z \gamma}$ also vanish. The wrong-sign Yukawa couplings for the down-type fermions cannot be realized in the SM with only one Higgs doublet [7]: The Higgs sector should be extended as in the two-Higgsdoublet model (2HDM) [8-12]. Reference [6] showed that the type-II $2 \mathrm{HDM}$ with a sequential fourth generation satisfies not only the Higgs signal strength measurements but also the oblique parameters $\Delta S$ and $\Delta T$. Follow-up studies focused on top-quark dipole moments [13], dark matter [14], and lepton flavor changing in the Higgs boson decays [15].

Despite the appeal of the model in satisfying the Higgs precision constraint through such a simple remedy, this model has a potentially dangerous spot, the phenomenology of the heavy Higgs bosons. The 2HDM has five physical Higgs bosons, the light $C P$-even scalar $h$, the heavy $C P$-even scalar $H$, the $C P$-odd pseudoscalar $A$, and 
two charged Higgs bosons $H^{ \pm}$. With a sequential fourth generation, the Yukawa couplings of the fourth generation fermion $F$ with all the Higgs bosons are proportional to the fermion mass $M_{F}$, which is naturally very large. In addition, the down-type fermion Yukawa couplings with $H$ and $A, Y_{b^{\prime}}^{H / A}$, are proportional to $\tan \beta$, while the up-type fermion couplings are inversely proportional to $\tan \beta$, where $\tan \beta$ is the ratio of two vacuum expectation values (VEVs) of two Higgs doublet fields. The cancellation of $t^{\prime}$ and $b^{\prime}$ contributions to the $h g g$ coupling does not occur in the $H g g$ and Agg couplings. At the LHC, $H$ and $A$ can be copiously produced.

Another concern is from the exact wrong-sign limit, the key that allows the sequential fourth generation. It relates two mixing angles $\alpha$ and $\beta$ through $\alpha+\beta=\pi / 2$, where $\alpha$ is the mixing angle between $h$ and $H$. Unless $\tan \beta$ becomes very large, the exact wrong-sign limit cannot approach the alignment limit of $\sin (\beta-\alpha)=1$. Since too-large $\tan \beta$ breaks the perturbativity of $Y_{b^{\prime}}^{H / A}$, the exact wrong-sign limit brings about a significant deviation from the alignment limit. One important consequence is the different dependence of the scalar quartic couplings on the heavy Higgs boson masses from the alignment limit. As will be shown, this difference has a strong impact on the perturbative unitarity of scalar-scalar scattering, the upper bounds on the heavy Higgs boson masses. The decoupling limit cannot be achieved along with the exact wrong-sign limit. The model does not have a safety zone. We need a comprehensive study on the phenomenology of the model, including the heavy Higgs bosons, which is our main purpose.

This paper is organized as follows. In Sec. II, we review the type-II $2 \mathrm{HDM}$ with a sequential fourth generation in the exact wrong-sign limit. The Higgs coupling modifiers in this model are compared with those in the alignment limit, with special focus on $\kappa_{V}$. Section III deals with the theoretical constraints on the scalar sector: the boundedfrom-below potential, unitarity, perturbativity, and vacuum stability. Here we explicitly show that the dependence of $\lambda_{3}$ on $M_{H}$ is very different from that in the alignment limit. In Sec. IV, we study the indirect constraints from the electroweak oblique parameters and the Higgs precision data. The observed $\kappa_{V}$ restricts $\tan \beta$ significantly. Based on the narrowed parameter space from the theoretical and indirect constraints, we study the decay and production of $H$ and $A$ in Sec. V. Here we point out that above the $b^{\prime} \bar{b}^{\prime}$ threshold, the total decay widths of both $H$ and $A$ become wide like $\Gamma_{\text {tot }}^{H / A} \sim M_{H / A}$. The ordinary analysis based on $\sigma \times \mathcal{B}$ does not work here. We suggest a method to probe this very broad resonance, not relying on the total event counting. The production cross sections of the gluon fusion of $H$ and $A$ are also studied. In Sec. VI, we consider direct search results for new particles at the LEP and LHC, $e^{+} e^{-} \rightarrow 4 b$, $4 \tau, 2 b 2 \tau$ and $p p \rightarrow \tau \tau, Z Z, Z h$. Two smoking-gun signals, $Z h$ for $A$ and $Z Z$ for $H$, are elaborated, drawing a rather strong conclusion that this model at leading order is excluded by the combination of theoretical and experimental constraints. In Sec. VII, we summarize and conclude.

\section{REVIEW OF THE 2HDM-SM4}

We consider a $2 \mathrm{HDM}$ with a sequential fourth generation in the exact wrong-sign limit. The Higgs sector and the fermion sector are extended by introducing two complex $S U(2)_{L}$ Higgs doublet scalar fields, $\Phi_{1}$ and $\Phi_{2}$ [16], and a sequential fourth generation, respectively:

$$
\begin{aligned}
\Phi_{i}= & \left(\begin{array}{c}
w_{i}^{+} \\
\frac{v_{i}+h_{i}+i \eta_{i}}{\sqrt{2}}
\end{array}\right), \quad\left(\begin{array}{c}
t_{L}^{\prime} \\
b_{L}^{\prime}
\end{array}\right), \quad t_{R}^{\prime}, \quad b_{R}^{\prime}, \\
& \left(\begin{array}{c}
\nu_{L}^{\prime} \\
\tau_{L}^{\prime}
\end{array}\right), \quad \nu_{R}^{\prime}, \quad \tau_{R}^{\prime},
\end{aligned}
$$

where $i=1,2$, and $v_{1,2}$ is the nonzero $\operatorname{VEV~of~} \Phi_{1,2}$. Note that the anomaly cancellation condition [17,18] requires the existence of the fourth generation leptons. When parametrizing $t_{\beta}=v_{2} / v_{1}$, one linear combination $H_{1}=c_{\beta} \Phi_{1}+$ $s_{\beta} \Phi_{2}$ has nonzero VEV of $v=\sqrt{v_{1}^{2}+v_{2}^{2}}=246 \mathrm{GeV}$, which generates the electroweak symmetry breaking. Its orthogonal combination $H_{2}=-s_{\beta} \Phi_{1}+c_{\beta} \Phi_{2}$ acquires zero VEV. For simplicity of notation, we take $s_{x}=\sin x$, $c_{x}=\cos x$, and $t_{x}=\tan x$.

In order to avoid flavor changing neutral currents (FCNC) at tree level, a discrete $Z_{2}$ symmetry is imposed, under which $\Phi_{1} \rightarrow \Phi_{1}$ and $\Phi_{2} \rightarrow-\Phi_{2}[19,20] .{ }^{1}$ Then, the most general scalar potential with $C P$ invariance and softly broken $Z_{2}$ symmetry is

$$
\begin{aligned}
V_{\Phi}= & m_{11}^{2} \Phi_{1}^{\dagger} \Phi_{1}+m_{22}^{2} \Phi_{2}^{\dagger} \Phi_{2}-m_{12}^{2}\left(\Phi_{1}^{\dagger} \Phi_{2}+\text { H.c. }\right) \\
& +\frac{1}{2} \lambda_{1}\left(\Phi_{1}^{\dagger} \Phi_{1}\right)^{2}+\frac{1}{2} \lambda_{2}\left(\Phi_{2}^{\dagger} \Phi_{2}\right)^{2}+\lambda_{3}\left(\Phi_{1}^{\dagger} \Phi_{1}\right)\left(\Phi_{2}^{\dagger} \Phi_{2}\right) \\
& +\lambda_{4}\left(\Phi_{1}^{\dagger} \Phi_{2}\right)\left(\Phi_{2}^{\dagger} \Phi_{1}\right)+\frac{1}{2} \lambda_{5}\left[\left(\Phi_{1}^{\dagger} \Phi_{2}\right)^{2}+\text { H.c. }\right] .
\end{aligned}
$$

The $C P$ invariance requires all of the parameters to be real. Note that the soft $Z_{2}$ symmetry breaking parameter $m_{12}^{2}$ can be negative.

There are five physical Higgs bosons, the light $C P$-even scalar $h$, the heavy $C P$-even scalar $H$, the $C P$-odd pseudoscalar $A$, and two charged Higgs bosons $H^{ \pm}$. They are related with the weak eigenstates in Eq. (1) via

$$
\begin{aligned}
\left(\begin{array}{l}
h_{1} \\
h_{2}
\end{array}\right) & =\mathbb{R}(\alpha)\left(\begin{array}{l}
H \\
h
\end{array}\right), \quad\left(\begin{array}{l}
\eta_{1} \\
\eta_{2}
\end{array}\right)=\mathbb{R}(\beta)\left(\begin{array}{l}
z^{0} \\
A
\end{array}\right), \\
\left(\begin{array}{l}
w_{1}^{ \pm} \\
w_{2}^{ \pm}
\end{array}\right) & =\mathbb{R}(\alpha)\left(\begin{array}{l}
w^{ \pm} \\
H^{ \pm}
\end{array}\right),
\end{aligned}
$$

\footnotetext{
${ }^{1}$ Recently, it was proposed that the addition of an Abelian gauge group can accommodate the absence of tree-level FCNC [21].
} 
where $z^{0}$ and $w^{ \pm}$are the Goldstone bosons that will be eaten by the $Z$ and $W$ bosons, respectively. The rotation matrix $\mathbb{R}(\theta)$ is

$$
\mathbb{R}(\theta)=\left(\begin{array}{cc}
c_{\theta} & -s_{\theta} \\
s_{\theta} & c_{\theta}
\end{array}\right)
$$

We consider the normal scenario where the observed Higgs boson is the lighter $C P$-even $h$, although the other scenario with $M_{H}=125 \mathrm{GeV}$ is still allowed by the Higgs precision data $[22,23]$.

The Yukawa couplings are different according to the $Z_{2}$ parity of the fermions. We fix $Q_{L} \rightarrow Q_{L}$ and $L_{L} \rightarrow L_{L}$ under $Z_{2}$ symmetry, where $Q_{L}$ and $L_{L}$ are the left-handed quark and lepton doublets, respectively. Then, each righthanded fermion field only couples to one scalar doublet field. There are four different ways to assign the $Z_{2}$ symmetry on the right-handed fermion fields, leading to four different types in the 2HDM: type-I, type-II, type-X, and type-Y. We parametrize the Yukawa interactions with the neutral Higgs bosons as

$$
-\mathcal{L}_{\text {Yuk }}=\sum_{f} \frac{m_{f}}{v}\left(\kappa_{f} \bar{f} f h+\xi_{f}^{H} \bar{f} f H-i \xi_{f}^{A} \bar{f} \gamma_{5} f A\right) .
$$

Note that $\kappa_{f}$ is the Higgs coupling modifier, parametrizing the NP effects on the Higgs couplings:

$$
\kappa_{i}=\frac{g_{i i h}}{g_{i i h}^{\mathrm{SM}}} \equiv 1+\delta \kappa_{i} .
$$

While $\kappa_{f}$ 's are different according to the 2HDM type, $\kappa_{V}$ and $\xi_{V}\left(V=W^{ \pm}, Z\right)$ have the common leading order expressions

$$
\kappa_{V}=s_{\beta-\alpha}, \quad \xi_{V}=c_{\beta-\alpha} .
$$

Because the observed Higgs boson at a mass of $125 \mathrm{GeV}$ is very SM-like, the so-called alignment limit [24] is usually adopted in the 2HDM, defined by

$$
\begin{aligned}
\alpha & =\beta-\frac{\pi}{2} \quad(\text { alignment limit }) \\
\rightarrow \kappa_{u} & =\kappa_{d}=1, \quad \kappa_{V}=1, \quad \xi_{V}=0,
\end{aligned}
$$

where $u=t, t^{\prime}, \nu^{\prime}$ and $d=b, b^{\prime}, \tau, \tau^{\prime}$. With a sequential fourth generation, however, this alignment limit does not guarantee a SM-like Higgs boson because of the large contribution from the fourth generation fermions to the loop-induced couplings of the Higgs boson, especially to $\kappa_{g}$ :

$$
\kappa_{g}=\frac{\kappa_{t} A_{1 / 2}^{h}\left(\tau_{t}\right)+\sum_{F} \kappa_{F} A_{1 / 2}^{h}\left(\tau_{F}\right)}{A_{1 / 2}^{h}\left(\tau_{t}\right)},
$$

where $\tau_{f}=m_{h}^{2} / 4 m_{f}^{2}, F=t^{\prime}, b^{\prime}$, and the expression for the loop function $A_{1 / 2}^{h}(\tau)$ is given in Ref. [25]. It is known that $A_{1 / 2}^{h}\left(\tau_{f}\right)$ approaches the value of $4 / 3$ when $m_{f} \gg m_{h}$. In the alignment limit $\left(\kappa_{t^{\prime}}=\kappa_{b^{\prime}}=1\right)$ with $M_{F} \gg m_{h}$, therefore, the value of $\kappa_{g}$ approaches 3 . We conclude that a sequential fourth generation in the SM or the aligned 2HDM is excluded by the Higgs precision data.

Based on the observation that $\delta \kappa_{g}$ is proportional to $\left(\kappa_{t^{\prime}}+\kappa_{b^{\prime}}\right)$ for $M_{F} \gg m_{h}$ and the current LHC data cannot determine the sign of $\kappa_{b}$ yet, the exact wrong-sign limit $[6,11,26]$ is suggested, given by

$$
\begin{aligned}
\alpha & =\frac{\pi}{2}-\beta(\text { exactwrong }- \text { sign limit }) \\
\rightarrow \kappa_{u} & =1, \quad \kappa_{d}=-1, \quad \kappa_{V}=\frac{t_{\beta}^{2}-1}{t_{\beta}^{2}+1}, \quad \xi_{V}=\frac{2 t_{\beta}}{t_{\beta}^{2}+1} .
\end{aligned}
$$

The wrong-sign Yukawa couplings for the down-type fermions cannot be realized in the SM where there exists only one scalar doublet field: all of the Yukawa couplings can be set positive by chiral rotation. We need an additional Higgs doublet field, which can be minimally realized in the 2HDM. Among four types of the 2HDM, only type-II can accommodate the exact wrong-sign limit where both $b^{\prime}$ and $\tau^{\prime}$ have opposite Yukawa couplings to $t^{\prime}$ and $\nu^{\prime}$. In what follows, 2HDM-SM4 denotes the type-II 2HDM with a sequential fourth generation in the exact wrong-sign limit.

A more surprising feature of the exact wrong-sign limit is that new contributions from the sequential fourth generation fermions to $\kappa_{\gamma \gamma}$ and $\kappa_{Z \gamma}$ are also suppressed in the heavy $M_{F}$ limit [6]:

$$
\begin{aligned}
& \delta \kappa_{\gamma \gamma} \propto \sum_{f=t^{\prime}, b^{\prime}, \tau^{\prime}} Q_{f}^{2} N_{C^{\prime}}^{f} \kappa_{f}=0, \\
& \delta \kappa_{Z \gamma} \propto \sum_{f=t^{\prime}, b^{\prime}, \tau^{\prime}} Q_{f}\left(T_{3}^{f}\right)_{L} N_{C}^{f} \kappa_{f}=0,
\end{aligned}
$$

where $Q_{f}$ is the electric charge of the fermion $f, N_{C}^{f}$ is the color factor, and $\left(T_{3}^{f}\right)_{L}$ is the isospin projection of the lefthanded $f_{L}$.

In the 2HDM, however, there exist other Higgs bosons, $H, A$, and $H^{ \pm}$. The exact wrong-sign condition simplifies $\xi_{u, d}^{H, A}$, defined in Eq. (5), into

$$
\xi_{u}^{H}=\xi_{u}^{A}=\frac{1}{t_{\beta}} \equiv \xi_{u}, \quad \xi_{d}^{H}=\xi_{d}^{A}=t_{\beta} \equiv \xi_{d} .
$$

An immediate concern is that the Yukawa couplings of $b^{\prime}$ and $\tau^{\prime}$ with $H$ and $A$, proportional to the heavy fermion masses, can be dangerously large, especially in the large $t_{\beta}$ limit. Theoretical principles and collider experiments associated with $H$ and $A$ constrain the model significantly. 
We now specify the model parameters in the 2HDMSM4. In the scalar potential sector, there are seven free parameters of $m_{12}^{2}, t_{\beta}$, and $\lambda_{1, \ldots, 5}$, after applying the tadpole conditions for $m_{11}$ and $m_{22}$. Equivalently, we can take the physical parameters of $m_{h}, M_{H}, M_{A}, M_{H^{ \pm}}, m^{2}, \alpha$ and $\beta$, where $m^{2}=m_{12}^{2} /\left(s_{\beta} c_{\beta}\right)$ is chosen because of its efficiency to show the invariance under the reparametrization in the space of the Lagrangian [27]. Since $m_{h}=125 \mathrm{GeV}$ is known and the exact wrong-sign limit relates $\alpha$ and $\beta$ as $\alpha+\beta=\pi / 2$, there are five free parameters in the scalar sector. In the fourth generation fermion sector, only their masses are unknown because their gauge and Yukawa couplings are the same as the SM fermions. In summary, the 2HDM-SM4 has the following model parameters:

$$
\begin{aligned}
& t_{\beta}, m^{2}, \quad M_{H}, \quad M_{A}, \quad M_{H^{ \pm}}, \quad M_{t^{\prime}}, \\
& M_{b^{\prime}}, \quad M_{\tau^{\prime}}, \quad M_{\nu^{\prime}} .
\end{aligned}
$$

Some brief comments on the fourth generation fermion masses $M_{F}$ and $M_{H^{ \pm}}$are in order here. For $M_{F}$, there are two kinds of constraints working in opposite ways, one from the unitarity and the other from direct searches. First, the perturbative unitarity for the fermion-fermion scattering sets the upper mass bound as $m_{q^{\prime}} \lesssim 550 \mathrm{GeV}$ and $m_{\ell^{\prime}} \lesssim$ $1.2 \mathrm{TeV}[28,29]$. On the other hand, direct searches for $t^{\prime}$ and $b^{\prime}$ at the LHC set the lower bounds of $M_{t^{\prime}} \gtrsim 680 \mathrm{GeV}$, under the assumption that the produced $t^{\prime}$ and $b^{\prime}$ decay into a SM quark accompanied by a $W$ or $Z$ boson [30]. If the mixing between the SM quarks and the fourth generation quarks is extremely small like $V_{i 4} \lesssim 10^{-7}$, however, no limits can be set [30]. The CDF Collaboration took the assumption of specific flavor-mixing rates and put the lower bound on $M_{t^{\prime}}, M_{b^{\prime}} \gtrsim 335-385 \mathrm{GeV}[31,32]$. With general flavor mixing, the lower mass bounds were recalculated to be as low as $290 \mathrm{GeV}[33,34]$. The fourth generation leptons have weaker bounds as $m_{\tau^{\prime}}>$ $100.8 \mathrm{GeV}$ and $m_{\nu^{\prime}}>41 \mathrm{GeV}$ [35]. The charged Higgs boson mass in a type-II $2 \mathrm{HDM}$ is most strongly constrained by the FCNC process $\bar{B} \rightarrow X_{s} \gamma$. The updated next-to-nextto-leading order SM prediction of $\mathcal{B}_{\mathrm{SM}}\left(\bar{B} \rightarrow X_{s} \gamma\right)[36,37]$ and the recent Bell result [38] get closer, yielding $M_{H^{ \pm}}>$ $570(440) \mathrm{GeV}$ for $t_{\beta} \gtrsim 2$ at $95 \%$ (99\%) C.L. For $t_{\beta} \lesssim 2$, the lower bound on $M_{H^{ \pm}}$increases significantly. Note that the fourth generation quarks do not affect the process $\bar{B} \rightarrow X_{s} \gamma$ under the assumption of $V_{4 i} \lesssim 10^{-7}$.

\section{THEORETICAL CONSTRAINTS ON THE SCALAR POTENTIAL}

The quartic coupling constants in the scalar potential $V_{\Phi}$ can be rewritten in terms of the physical mass parameters, which are in the exact wrong-sign limit

$$
\begin{aligned}
& \lambda_{1}=\frac{1}{v^{2}}\left[t_{\beta}^{2}\left(M_{H}^{2}-m^{2}\right)+m_{h}^{2}\right], \\
& \lambda_{2}=\frac{1}{v^{2}}\left[\frac{1}{t_{\beta}^{2}}\left(M_{H}^{2}-m^{2}\right)+m_{h}^{2}\right], \\
& \lambda_{3}=\frac{1}{v^{2}}\left[-m^{2}+M_{H}^{2}-m_{h}^{2}+2 M_{H^{ \pm}}^{2}\right], \\
& \lambda_{4}=\frac{1}{v^{2}}\left[m^{2}+M_{A}^{2}-2 M_{H^{ \pm}}^{2}\right], \\
& \lambda_{5}=\frac{1}{v^{2}}\left[m^{2}-M_{A}^{2}\right] .
\end{aligned}
$$

They are constrained by the following theoretical conditions:

(1) The scalar potential $V_{\Phi}$ should be bounded from below in any direction, requiring $[39,40]$

$$
\begin{aligned}
& \lambda_{1}>0, \quad \lambda_{2}>0, \quad \lambda_{3}>-\sqrt{\lambda_{1} \lambda_{2}}, \\
& \lambda_{3}+\lambda_{4}-\left|\lambda_{5}\right|>-\sqrt{\lambda_{1} \lambda_{2}} .
\end{aligned}
$$

(2) The tree-level perturbative unitarity demands [41-43]

$$
\left|a_{i, \pm}\right| \leq 1
$$

where $a_{i, \pm}(i=1, \ldots, 6)$ are the eigenvalues of the $T$ matrix for the $S$-wave amplitudes of the scalar-scalar scattering, given by

$$
\begin{gathered}
a_{1, \pm}=\frac{1}{32 \pi}\left[3\left(\lambda_{1}+\lambda_{2}\right) \pm \sqrt{9\left(\lambda_{1}-\lambda_{2}\right)^{2}+4\left(2 \lambda_{3}+\lambda_{4}\right)^{2}}\right] \\
a_{2, \pm}=\frac{1}{32 \pi}\left[\lambda_{1}+\lambda_{2} \pm \sqrt{\left(\lambda_{1}-\lambda_{2}\right)^{2}+4 \lambda_{4}^{2}}\right] \\
a_{3, \pm}=\frac{1}{32 \pi}\left[\lambda_{1}+\lambda_{2} \pm \sqrt{\left(\lambda_{1}-\lambda_{2}\right)^{2}+4 \lambda_{5}^{2}}\right] \\
a_{4, \pm}=\frac{1}{16 \pi}\left(\lambda_{3}+2 \lambda_{4} \pm 3 \lambda_{5}\right) \\
a_{5, \pm}=\frac{1}{16 \pi}\left(\lambda_{3} \pm \lambda_{4}\right) \\
a_{6, \pm}=\frac{1}{16 \pi}\left(\lambda_{3} \pm \lambda_{5}\right) .
\end{gathered}
$$

(3) The perturbativity of scalar quartic couplings requires

$$
\left|\lambda_{i}\right|<4 \pi, \quad i=1, \ldots, 5 .
$$

(4) The vacuum of $V_{\Phi}$ should be global, which happens if and only if [44]

$$
D=m_{12}^{2}\left(m_{11}^{2}-k^{2} m_{22}^{2}\right)\left(t_{\beta}-k\right)>0,
$$

where $k=\left(\lambda_{1} / \lambda_{2}\right)^{1 / 4}$. 

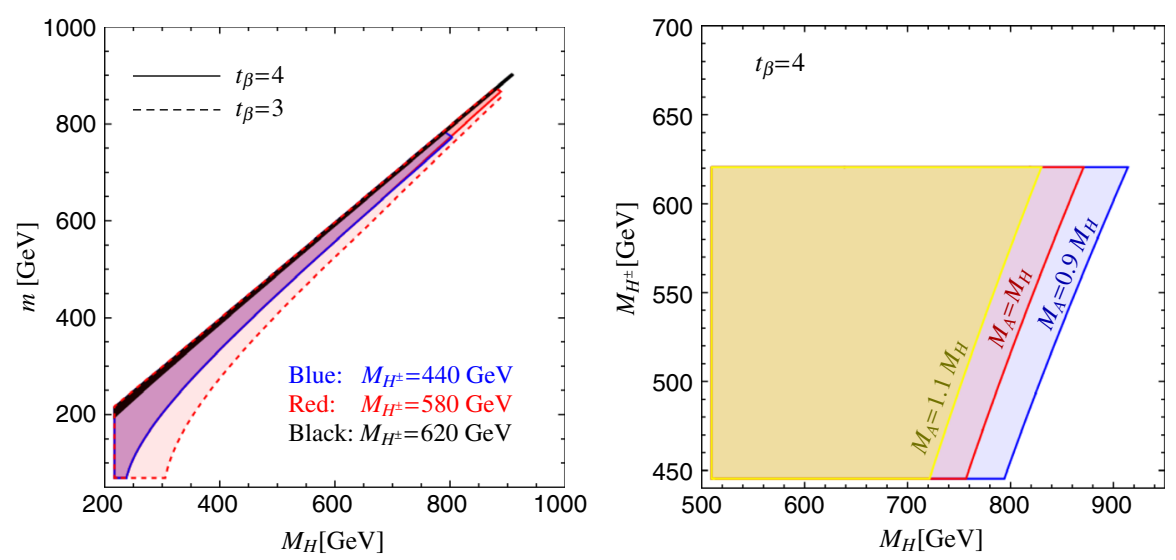

FIG. 1. Left panel: Theoretically allowed parameter space of $\left(M_{H}, m\right)$ for $t_{\beta}=3,4, M_{H^{ \pm}}=440,580,620 \mathrm{GeV}$, and $M_{A}=0.9 M_{H}$. Right panel: Theoretically allowed parameter space of $\left(M_{H}, M_{H^{ \pm}}\right)$for $t_{\beta}=4$ and $M_{A}=(1 \pm 0.1) M_{H}$.

In the exact wrong-sign limit, the theoretical constraints are more difficult to satisfy than in the alignment limit. Crucial is $\lambda_{3}$, which shows different dependence on $M_{H}$ :

$$
\lambda_{3}= \begin{cases}\frac{1}{v^{2}}\left[2 M_{H^{ \pm}}^{2}+M_{H}^{2}-m_{h}^{2}-m^{2}\right] & \text { in the exact wrong-sign limit } \\ \frac{1}{v^{2}}\left[2 M_{H^{ \pm}}^{2}-M_{H}^{2}+m_{h}^{2}-m^{2}\right] & \text { in the alignment limit. }\end{cases}
$$

In both limits, $\lambda_{3}$ has the same dependence on $M_{H^{ \pm}}^{2}$ but opposite signs for the $M_{H}^{2}$ terms. Since $M_{H^{ \pm}}$in a type-II 2HDM should be very heavy to explain the $b \rightarrow s \gamma$ result, heavy $M_{H}$ in the exact wrong-sign limit should easily increase $\lambda_{3}$ above $4 \pi$. In the alignment limit, the negative $M_{H}^{2}$ contribution cancels the positive $M_{H^{ \pm}}^{2}$ contribution to some extent since it is able to control the $\lambda_{3}$ value.

Figure 1 presents the theoretically allowed parameter space. In the left panel, we show the allowed parameter space $^{2}$ of $\left(M_{H}, m\right)$ for $M_{H^{ \pm}}=440 \mathrm{GeV}$ and $t_{\beta}=4$ (blue region), $M_{H^{ \pm}}=580 \mathrm{GeV}$ and $t_{\beta}=3,4$ (red region), and $M_{H^{ \pm}}=620 \mathrm{GeV}$ and $t_{\beta}=4$ (black region). We also set $M_{A}=0.9 M_{H}$ in order to explain the oblique $\Delta T$ parameter. Two cases of $t_{\beta}=3$ and $t_{\beta}=4$ for the same $M_{H^{ \pm}}=$ $580 \mathrm{GeV}$ show that small $t_{\beta}$ allows more freedom for $m$, but it does not significantly change the allowed range for $M_{H}$. When $t_{\beta}$ is very large, only a fine linear line along $M_{H}=m$ is allowed, irrespective of $M_{H^{ \pm}}$. The most important result is that theoretical constraints put an upper bound on $M_{H}$, of which the $M_{H^{ \pm}}$dependence is small and the $t_{\beta}$ dependence is negligible.

The charged Higgs boson mass is also bounded from above. In the right panel of Fig. 1, we show the theoretically allowed parameter space of $\left(M_{H}, M_{H^{ \pm}}\right)$for $t_{\beta}=4$. We consider three cases of $M_{A}=M_{H}, M_{A}=1.1 M_{H}$, and

\footnotetext{
${ }^{2}$ Since only positive $m^{2}$ is allowed by the theoretical constraints, $m=\sqrt{m^{2}}$ is presented.
}

$M_{A}=0.9 M_{H}$ to explain the oblique parameter $\Delta T$. It can be seen that $M_{H}$ cannot exceed about $920 \mathrm{GeV}$ and $M_{H^{ \pm}}$should be smaller than $620 \mathrm{GeV}$. Since the $t_{\beta}$ dependence on the upper bounds is negligible, as shown in the left panel of Fig. 1, the presence of the upper bounds on $M_{H}, m$, and $M_{H^{ \pm}}$is a generic feature of the 2HDMSM4. We find that relaxing the degeneracy of $M_{H}$ and $M_{A}$ does not change the results much: The upper bound on $M_{H}$ is about $945 \mathrm{GeV}$ when $M_{A} \simeq 0.85 M_{H}$. This result is unexpected. In the so-called normal mass hierarchy scenario where the observed $125-\mathrm{GeV}$ scalar is the lighter $h$, it is usually expected that the theory can hide in the decoupling region. However, the exact wrong-sign limit does not allow decoupling of the theory. This is a unique feature of this model.

\section{INDIRECT CONSTRAINTS FROM OBLIQUE PARAMETERS AND HIGGS PRECISION DATA}

In this section we narrow down the parameter space by applying the indirect constraints from the electroweak oblique parameters and the Higgs precision data.

\section{A. Electroweak oblique parameters $\Delta S$ and $\Delta T$}

The oblique parameters $\Delta S$ and $\Delta T$ are affected by the sequential fourth generation fermions $[28,45]$ as well as by new scalar bosons $[46,47]$. The current experimental data are consistent with the SM values, given by [35] 


$$
\Delta S=0.05 \pm 0.10, \quad \Delta T=0.08 \pm 0.12
$$

where the parameter $\Delta T$ is more sensitive to new particles. It is well known that new contributions to $\Delta T$ are suppressed when the new particles running in the self-energy diagrams of gauge bosons have the same masses. We require that $M_{t^{\prime}} \simeq M_{b^{\prime}}, M_{\tau^{\prime}} \simeq M_{\nu^{\prime}}$, and two masses among $M_{H}, M_{A}$, and $M_{H^{ \pm}}$are degenerate. We also note that the contributions from $H$ and $A$ to the oblique parameter $\Delta T$ are negative [16], while those from the fourth generation fermions are positive [28,45,47]. Large mass splittings in the scalar and fourth generation fermion sectors are allowed if exquisite cancellation occurs. In this work, however, we do not consider the conspiracy between the new scalar sector and the new fermion sector in explaining the oblique parameters.

\section{B. Higgs precision data}

We take the combined analysis of ATLAS and CMS on the Higgs coupling modifier $\kappa_{i}$ based on the LHC run 1 data, corresponding to integrated luminosities per experiment of $5 \mathrm{fb}^{-1}$ at $\sqrt{s}=7 \mathrm{TeV}$ and $20 \mathrm{fb}^{-1}$ at $\sqrt{s}=8 \mathrm{TeV}$ [48]. ${ }^{3}$ The analysis is based on a few assumptions, each of which constrains $\kappa_{i}$ 's differently. The 2HDM-SM4 model belongs to the category where new loop couplings beyond the SM (BSM) are allowed. The analysis result is that all $\kappa_{i}$ 's are consistent with the SM value. For the allowed values of $\kappa_{i}$ 's in this category, we refer the reader to Fig. 15 in Ref. [48].

The exact wrong-sign limit naturally explains the SMlike $\kappa_{t},\left|\kappa_{b, \tau}\right|, \kappa_{g}$ and $\kappa_{\gamma}$. One minor concern is $\left|\kappa_{b}\right|$, of which the maximum value at $2 \sigma$ level is about $5 \%$ smaller than 1 . Since a small deviation from the exact wrong-sign limit can easily accommodate this result, we stick to the exact wrong-sign limit as our reference point. On the other hand, $\kappa_{V}$ can significantly deviate from 1. In Fig. 2, we show $\kappa_{V}$ and $\xi_{V}$ as a function of $t_{\beta}$. The observed $\kappa_{V}$ requires very large $t_{\beta}$ : If $\kappa_{V}=0.98$, we need $t_{\beta} \approx 9.95$. However, toolarge $t_{\beta}$ violates the perturbativity of the Yukawa couplings of $b^{\prime}$ and $\tau^{\prime}$ with $H$ and $A, Y_{b^{\prime}, \tau^{\prime}}^{H / A}=t_{\beta} M_{b^{\prime}, \tau^{\prime}} / v$. If $\kappa_{V}=0.98$ and $M_{b^{\prime}}=340 \mathrm{GeV}$, e.g., we have $Y_{b^{\prime}}^{H}=13.8$. In summary, there is tension on the value of $t_{\beta}$ : The observed $\kappa_{V}$ pushes $t_{\beta}$ upward; the perturbativity of the $H b^{\prime} \bar{b}^{\prime}$ coupling presses $t_{\beta}$ downward.

In Fig. 3, we summarize the constraints from the oblique parameter $\Delta T$ at $2 \sigma$ level, the Higgs coupling modifier $\kappa_{V}$ at $2 \sigma$ level, and the perturbativity of $Y_{b^{\prime}}^{H / A}$. Here $\Delta M_{D U}=$ $M_{b^{\prime}}-M_{t^{\prime}}=M_{\tau^{\prime}}-M_{\nu^{\prime}}$ and $M_{U}\left(=M_{t^{\prime}}=M_{\nu^{\prime}}\right)$ is fixed to be $300 \mathrm{GeV}$. The observed $\kappa_{V}$ allows $t_{\beta} \gtrsim 3$, while the

\footnotetext{
${ }^{3}$ More recent analyses of the $13-\mathrm{TeV}$ data $[49,50]$ are neither combined ones of the ATLAS and CMS nor suitable for $\kappa_{t}=-\kappa_{b}$.
}

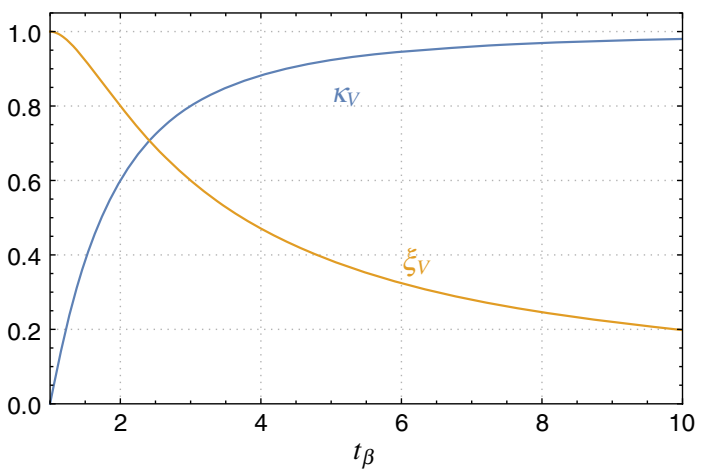

FIG. 2. $\kappa_{V}$ and $\xi_{V}$ as a function of $t_{\beta}$.

perturbativity of $Y_{b^{\prime}, \tau^{\prime}}^{H / A}$ requires $t_{\beta} \lesssim 8$. The oblique parameter $\Delta T$ demands that the mass of an up-type fourth generation fermion be similar to that of the corresponding down-type fermion as $\Delta M / M \lesssim 20 \%$.

In addition, the experimental result on exotic Higgs decay $\mathcal{B}_{\mathrm{BSM}}^{h} \leq 0.34$ [48] has an important implication on the mass of the pseudoscalar $A$. In the $2 \mathrm{HDM}, M_{A}$ is a free parameter, so $A$ can be light enough to kinematically allow $h \rightarrow A A$. The observed oblique parameter $\Delta T$ can be explained by the mass degeneracy $M_{H} \simeq M_{H^{ \pm}}$. When writing $\mathcal{L} \supset \lambda_{h A A} A A h / 2$, the partial decay rate is

$$
\Gamma(h \rightarrow A A)=\frac{1}{32 \pi m_{h}} \lambda_{h A A}^{2} \sqrt{1-\frac{4 M_{A}^{2}}{m_{h}^{2}}} .
$$

In the general 2HDM, $\lambda_{h A A}$ is unknown for the given $m_{h}$ and $M_{A}$ because of an additional free parameter $m^{2}$. In the

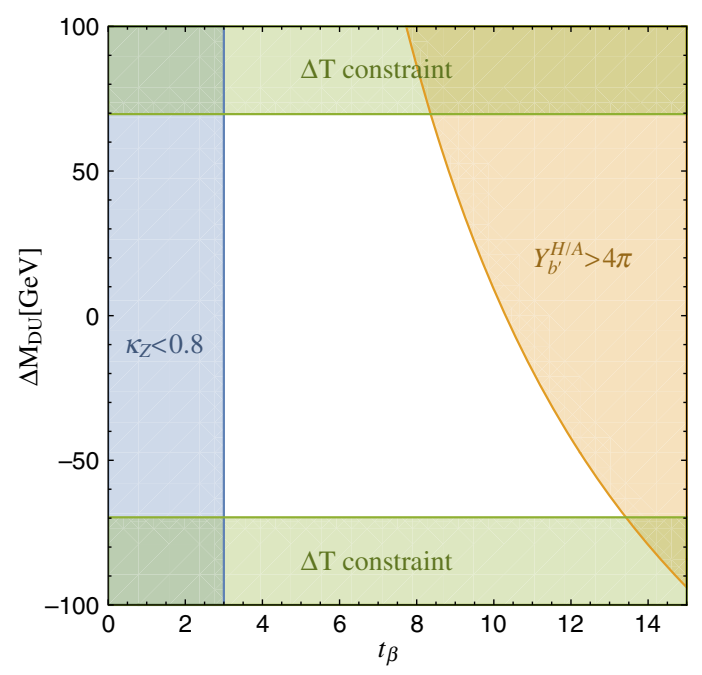

FIG. 3. The excluded regions of $\left(t_{\beta}, \Delta M_{D U}\right)$ by the observed Higgs coupling modifier $\kappa_{V}$ at $2 \sigma$ level, the oblique parameter $\Delta T$ at $2 \sigma$ level, and the perturbativity of $Y_{b^{\prime}}^{H / A}$. Here $\Delta M_{D U}=M_{b^{\prime}}-$ $M_{t^{\prime}}=M_{\tau^{\prime}}-M_{\nu^{\prime}}$ for the fixed $M_{U}=300 \mathrm{GeV}$. For the oblique $\Delta T$ parameter, we set $M_{H}=M_{A}=M_{H^{ \pm}}=600 \mathrm{GeV}$. 
exact wrong-sign limit $(\alpha+\beta=\pi / 2)$, however, the $m^{2}$ term is proportional to $c_{\beta+\alpha}$ and thus vanishes. The value of $\lambda_{h A A}$ is determined by $t_{\beta}$ and $M_{A}$ as

$$
\lambda_{h A A}=\frac{c_{2 \beta}}{v}\left[2 M_{A}^{2}-m_{h}^{2}\right] .
$$

The observed $\mathcal{B}_{\mathrm{BSM}}^{h} \leq 0.34$ is translated into $\Gamma(h \rightarrow A A) \lesssim$ $\Gamma_{\text {tot }}^{h, \mathrm{SM}} / 2$, which can be satisfied when $0.9 \lesssim t_{\beta} \lesssim 1.1$ $\left(0.62 \lesssim t_{\beta} \lesssim 1.60\right)$ for $M_{A} \ll m_{h}\left(M_{A}=62 \mathrm{GeV}\right)$. This small $t_{\beta}$ region is excluded by the observed $\kappa_{V}$ (see Fig. 2). In summary, a light pseudoscalar boson with $M_{A} \lesssim m_{h} / 2$ in the 2HDM-SM4 is excluded by the observed $\mathcal{B}_{\mathrm{BSM}}^{h}$.

\section{DECAY AND PRODUCTION OF $H$ AND $A$}

\section{A. Decays}

In this section, we discuss the decay and production of neutral heavy Higgs bosons, $H$ and $A$. Considering the theoretical and direct search bounds on $M_{F}$, the perturbative unitarity of the scalar-scalar scattering, and the $b \rightarrow s \gamma$ constraint altogether, we take the following benchmark scenario:

$$
\begin{aligned}
m & =M_{H}, \quad M_{A} \approx M_{H}, \quad M_{H^{ \pm}}=580 \mathrm{GeV}, \\
M_{t^{\prime}} & =300 \mathrm{GeV}, \quad M_{b^{\prime}}=340 \mathrm{GeV}, \\
M_{\nu^{\prime}} & =430 \mathrm{GeV}, \quad M_{\tau^{\prime}}=380 \mathrm{GeV} .
\end{aligned}
$$

Kinematically, the decays of $H \rightarrow A A$ and $H \rightarrow H^{+} H^{-}$ are prohibited. Note that the decays of $H \rightarrow W^{ \pm} H^{\mp}$ and $A \rightarrow Z h$ are possible through the interaction Lagrangian of

$$
\mathcal{L} \supset \frac{g_{Z}}{2} c_{\beta-\alpha} Z_{\mu} h \stackrel{\leftrightarrow}{\partial^{\mu}} A+i \frac{g}{2} s_{\beta-\alpha}\left[W_{\mu} H \stackrel{\leftrightarrow}{\partial^{\mu}} H^{-}+\text {H.c. }\right]
$$

where $g_{Z}=g / c_{\theta_{W}}, \theta_{W}$ is the weak mixing angle, and $\phi \overleftrightarrow{\partial}^{\mu} \eta=\phi \partial^{\mu} \eta-\left(\partial^{\mu} \phi\right) \eta$.

In Fig. 4, we show the branching ratios of $H$ as a function of $M_{H}$ for $t_{\beta}=4$ and $m=M_{H}$. Before the $W W$ threshold,

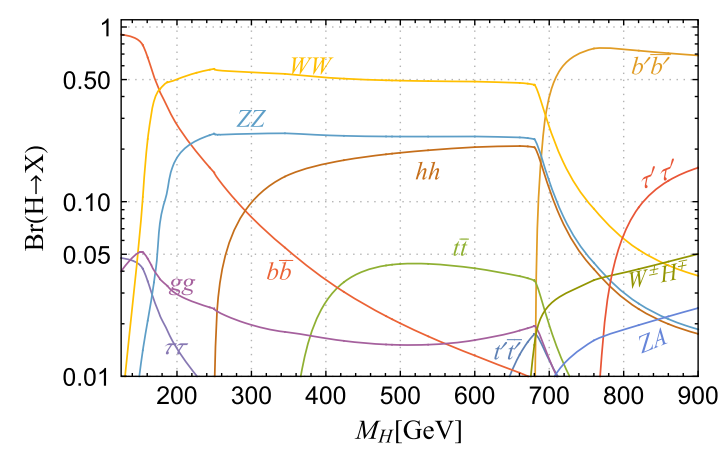

FIG. 4. The branching ratios of $H$ as a function of $M_{H}$ for $t_{\beta}=4$. We set $M_{t^{\prime}}=300 \mathrm{GeV}, \quad M_{b^{\prime}}=340 \mathrm{GeV}, M_{\nu^{\prime}}=430 \mathrm{GeV}$, $M_{\tau^{\prime}}=380 \mathrm{GeV}, m=M_{H}$, and $M_{A}=M_{H^{ \pm}}=580 \mathrm{GeV}$. the dominant decay mode of $H$ is into $b \bar{b}$, followed by $\tau \tau$ and $g g$ modes because of the $t_{\beta}$ enhancement in $Y_{b, \tau}^{H}$. After the $W W / Z Z$ threshold, nonzero $\xi_{V}=c_{\beta-\alpha}\left(c_{\beta-\alpha} \approx 0.47\right.$ for $t_{\beta}=4$ ) yields dominant decay of $H$ into $W W$ and $Z Z$ because of the longitudinal polarization enhancement in the heavy scalar decay into a massive gauge boson pair [51].

For $M_{H}>2 m_{h}$, the decay of $H \rightarrow h h$ also becomes important. The triple Higgs coupling $\lambda_{H h h}$ in the exact wrong-sign limit is

$$
\lambda_{H h h}=\frac{c_{\beta-\alpha}}{v}\left(m^{2}-\frac{1}{2} M_{H}^{2}-m_{h}^{2}\right)
$$

With the sizable $c_{\beta-\alpha}$ and the condition of $m \simeq M_{H}$ from the theoretical constraints shown in Fig. 1, the branching ratio of $H \rightarrow h h$ is substantial. Above the $t \bar{t}$ threshold, the $H \rightarrow t \bar{t}$ mode turns on, but not dominantly because the $H t \bar{t}$ coupling is inversely proportional to $t_{\beta}$. For the same reason, the $H \rightarrow t^{\prime} \bar{t}^{\prime}$ mode is also minor even after the $t^{\prime} \bar{t}^{\prime}$ threshold. After the $b^{\prime} \bar{b}^{\prime}$ and $\tau^{\prime} \tau^{\prime}$ threshold, $H \rightarrow b^{\prime} \bar{b}^{\prime}$ is dominant and $H \rightarrow \tau^{\prime} \tau^{\prime}$ is the second dominant. The third dominant decay channel is into $H \rightarrow W^{ \pm} H^{\mp}$, which always remains important because its vertex is proportional to $s_{\beta-\alpha}$ [see Eq. (26)].

The branching ratios of the $C P$-odd Higgs boson $A$ as a function of $M_{A}$ are shown in Fig. 5. We take the benchmark scenario in Eq. (25). Because of the $C P$-odd nature, $A$ does not decay into $W W, Z Z$, or $h h$, but it does decay into $Z h$ since the $A Z h$ vertex is proportional to non-negligible $c_{\beta-\alpha}$. Before the $Z h$ threshold, the $b \bar{b}$ mode is dominant: The $A b \bar{b}$ coupling also has $t_{\beta}$ enhancement. The second dominant decay mode into $g g$ has a much larger branching ratio than the $\tau \tau$ mode, unlike the case of $H$. This is because of the larger $g g$ loop function for the $C P$-odd scalar than that for the $C P$-even scalar. When $m_{Z}+m_{h}<M_{A}<2 M_{b^{\prime}}$, the decay of $A \rightarrow Z h$ is dominant, contrary to the alignment limit. In this mass range, the decay into $g g$ is still important. We expect a sizable cross section of gluon fusion production of $A$. The branching ratios of the $t \bar{t}$ and $t^{\prime} \bar{t}^{\prime}$ modes are

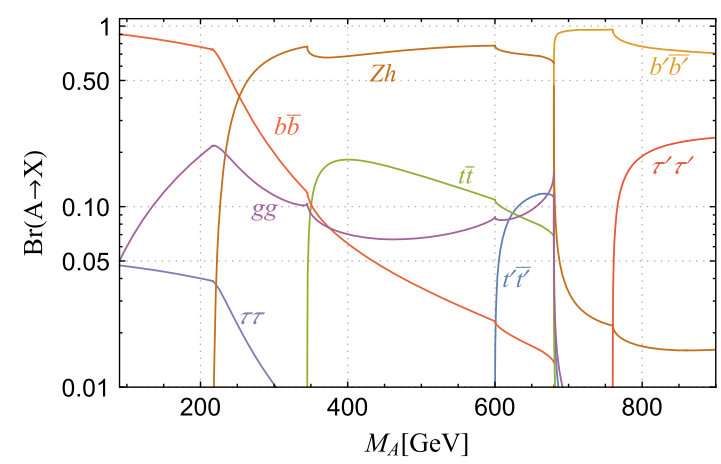

FIG. 5. The branching ratios of $A$ as a function of $M_{A}$ for $t_{\beta}=4$. We set $M_{t^{\prime}}=300 \mathrm{GeV}, M_{b^{\prime}}=340 \mathrm{GeV}, M_{\nu^{\prime}}=430 \mathrm{GeV}$, $M_{\tau^{\prime}}=380 \mathrm{GeV}$, and $m=M_{H}=M_{H^{ \pm}}=580 \mathrm{GeV}$. 
not as large as that of the $Z h$ mode because both $A t \bar{t}$ and $A t^{\prime} \bar{t}^{\prime}$ couplings are inversely proportional to $t_{\beta}$. After the $b^{\prime} \bar{b}^{\prime}$ and $\tau^{\prime} \tau^{\prime}$ threshold, $A$ decays into $b^{\prime} \bar{b}^{\prime}$ dominantly, followed by the $\tau^{\prime} \tau^{\prime}$ mode. The next dominant mode is into $Z h$ with $\mathcal{B}(A \rightarrow Z h) \sim \mathcal{O}(1) \%$.

In Fig. 6, we show the total decay widths of $H$ and $A$ as a function of $M_{H}$ and $M_{A}$ for $t_{\beta}=3$ and $t_{\beta}=4$ in the benchmark scenario. For both $H$ and $A, \Gamma_{\text {tot }}$ increases rapidly with its mass, especially above the $b^{\prime} \bar{b}^{\prime}$ threshold. The dependence of $\Gamma_{\text {tot }}^{H / A}$ on $t_{\beta}$ is interesting. Before the $b^{\prime} \bar{b}^{\prime}$ threshold, the larger $t_{\beta}$ is, the smaller the total decay width is. This behavior is due to the fact that the major decay modes before the $b^{\prime} \bar{b}^{\prime}$ threshold, $H \rightarrow W W / Z Z$ and $A \rightarrow Z h$, have partial decay rates that are all proportional to $\xi_{V}^{2}$, which decreases with increasing $t_{\beta}$ (see Fig. 2). After the $b^{\prime} \bar{b}^{\prime}$ threshold, on the other hand, the dominant decay rate $\Gamma\left(H / A \rightarrow b^{\prime} \bar{b}^{\prime}\right)$ is proportional to $t_{\beta}^{2}$.

Another crucial question related to $\Gamma_{\text {tot }}$ is whether $H$ and $A$ can be probed as a narrow resonance so that the number of new events is proportional to the production cross section times the branching ratio. For reference, the LHC criteria as a narrow resonance is $\Gamma / M \leq 1 \%$ in the $\gamma \gamma$ channel [52,53], $\Gamma / M \leq 0.5 \%$ in the $Z Z$ channel [49,54], and $\Gamma / M \leq 15 \%$ in the dijet channel [55]. When $t_{\beta}=4$, e.g., heavy Higgs bosons like $M_{H} \gtrsim$ $350 \mathrm{GeV}$ and $M_{A} \gtrsim 300 \mathrm{GeV}$ do not belong to the narrow width category, which requires going beyond $\sigma \times \mathcal{B}$. Particularly above the $b^{\prime} \bar{b}^{\prime}$ threshold, both $\Gamma_{\text {tot }}^{H}$ and $\Gamma_{\text {tot }}^{A}$ are large like $\Gamma_{\text {tot }} \sim M_{H}$. It is almost impossible to observe a mass peak in this mass region. We need a new strategy.

In order to deal with the very large width case, such as $\Gamma_{\text {tot }}^{H / A} \sim M_{H / A}$, two points should be considered. First, we need a full calculation of $\sigma(p p \rightarrow i j)$ including the SM continuum background in order not to miss the significant interference. The second point is that new events spread out over multiple $m_{i j}$ bins, not only to the bin of $m_{i j}=M$.

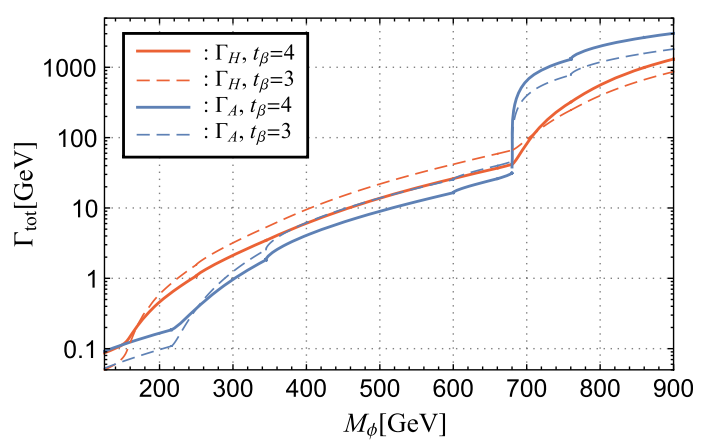

FIG. 6. Total decay width of $H$ and $A$ as a function of their masses for two $t_{\beta}$ cases, $t_{\beta}=3$ and $t_{\beta}=4$. We set $m=M_{H}$, $M_{t^{\prime}}=300 \mathrm{GeV}, \quad M_{b^{\prime}}=340 \mathrm{GeV}, \quad M_{\nu^{\prime}}=430 \mathrm{GeV}, \quad$ and $M_{\tau^{\prime}}=380 \mathrm{GeV}$, and do not include the decays of $H \rightarrow$ $H^{+} H^{-}$and $H \rightarrow A A$.
Without the possibility of observing a mass peak, we may rely on counting total events, which requires a very good understanding of the background. Or we can utilize the results of the usual analysis of the resonance searches for the excess in the invariant mass bins, which takes the following steps: (i) Events are collected in a specific $m_{i j}$ bin; (ii) the number of events in the bin is compared with that of the expected background; (iii) no excess leads to the upper bounds on $\sigma \times \mathcal{B}(X \rightarrow i j)$ for a possible new particle with mass $m_{i j}$. Since multiple $m_{i j}$ bins are affected by a single new particle when $\Gamma \sim M$, we need to calculate the excess of each $m_{i j}$ bin nearby $M$ and compare with the upper bounds on $\sigma \times \mathcal{B}(A \rightarrow Z h)$ for the corresponding bin. The size of each $m_{i j}$ bin, $\Delta M$, depends on the experimental analysis. Since our $d \sigma^{\mathrm{NP}}$ already includes the SM contributions, the excess over the SM backgrounds corresponds to the difference between the full $d \sigma^{\mathrm{NP}}$ and $d \sigma^{\mathrm{SM}}$. In order to compare with the excess in the $m_{i j} \in$ $[M, M+\Delta M]$ bin, therefore, we calculate the partially integrated cross section $\Delta \sigma^{\mathrm{NP}}$ given by

$\Delta \sigma^{\mathrm{NP}}=\int_{M}^{M+\Delta M} d m_{i j}\left[\frac{d \sigma^{\mathrm{NP}}(g g \rightarrow i j)}{d m_{i j}}-\frac{d \sigma^{\mathrm{SM}}(g g \rightarrow i j)}{d m_{i j}}\right]$.

We use this method when constraining very heavy $A$ and $H$ in the $Z h$ and $Z Z$ final states, respectively.

\section{B. Production}

We study the production of $H$ and $A$. The production cross section of $H / A$ with a small width is [56]

$$
\sigma(p p \rightarrow H / A)=\frac{1}{s M_{H / A}} \sum_{\mathfrak{p}} C_{\mathfrak{p p}} \Gamma(H / A \rightarrow \mathfrak{p p}),
$$

where $\mathfrak{p}$ is a parton in a proton and $C_{\mathfrak{p p}}$ is the $\mathfrak{p p}$ dimensionless partonic integral. Because $C_{b \bar{b}} / C_{g g} \sim 0.01$

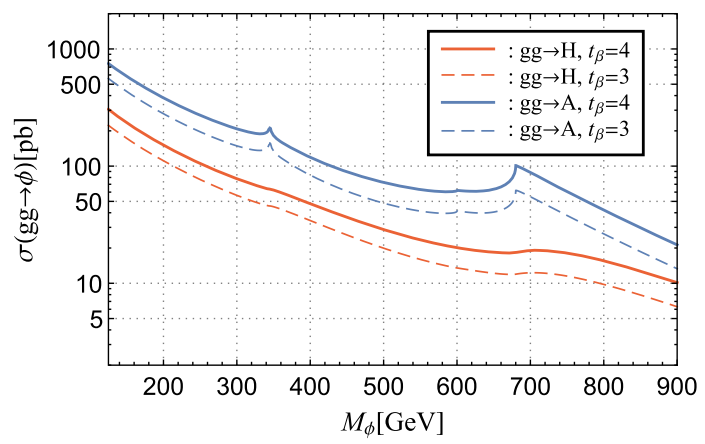

FIG. 7. The gluon fusion production cross sections of $H$ and $A$ as functions of $M_{H}$ and $M_{A}$ at the 13-TeV LHC for $t_{\beta}=3$ and $t_{\beta}=4$. We apply the NNLO $K$ factor to the heavy Higgs resonance production by using the HIGLU package [57]. 
at the $13-\mathrm{TeV}$ LHC and moderate $t_{\beta} \in[3,8]$ implies nottoo-large $H b \bar{b}$ and $A b \bar{b}$ couplings, we consider only the gluon fusion production. Figure 7 presents the gluon fusion production cross sections of $H$ and $A$ at the $13-\mathrm{TeV}$ LHC as a function of $M_{H}$ and $M_{A}$, respectively. We consider $t_{\beta}=3$ and $t_{\beta}=4$, and include the NNLO $K$ factor by using the HIGLU package [57]. In the whole mass range, the production cross section is large: For $M_{H}, M_{A}=600 \mathrm{GeV}$, e.g., $\sigma(p p \rightarrow H / A) \sim \mathcal{O}(10)$ pb. The pseudoscalar $A$ has a larger cross section than $H$ because of the larger loop function for $g g$. As $t_{\beta}$ increases, $\sigma(g g \rightarrow H / A)$ increases because the contribution from the $b^{\prime}$ quark in the loop is enhanced. For the $C P$-odd $A$, the threshold effects at $M_{A}=2 m_{t}$ and $M_{A}=2 M_{b^{\prime}}$ are more prominent due to the cusp structure of the real part of the $g g$ form factor $A_{1 / 2}^{A}(\tau)$ [58]. We caution the reader that the production cross section in Eq. (29) for $M_{H / A} \gtrsim 2 M_{b^{\prime}}$ gives just a rough estimation. When $\Gamma_{\text {tot }} \sim M_{H / A}$, the production of $H / A$ itself is not meaningful. We need to set the final states $i j$ and to perform the full calculation of $\sigma(p p \rightarrow i j)$, including the SM continuum background, because the interference effects crucially depend on the final state.

\section{CONSTRAINTS FROM DIRECT SEARCHES}

In this section, we study the constraints from the direct searches for neutral scalar bosons at high energy colliders. The allowed region for the charged Higgs boson mass, $570 \lesssim M_{H^{ \pm}} \lesssim 620 \mathrm{GeV}$, is very difficult to probe because of the extremely small production rate of $H^{ \pm}$[59]. Our main target channels are summarized in Table I. Brief comments on $\gamma \gamma, \mu^{+} \mu^{-}$, and $b \bar{b}$ modes are in order here. Although the very clean $\gamma \gamma$ mode is searched for from $m_{\gamma \gamma}=65(70) \mathrm{GeV}$ by the ATLAS (CMS) experiments $[60,61]$, it does not constrain the model since the branching ratio is very small: $\mathcal{B}(A \rightarrow \gamma \gamma) \sim \mathcal{O}\left(10^{-5}\right)$ for $M_{A}=65 \mathrm{GeV}$. The data in the $\mu^{+} \mu^{-}$channel [62] are also insufficient because of the extremely small $\mathcal{B}\left(H / A \rightarrow \mu^{+} \mu^{-}\right) \lesssim \mathcal{O}\left(10^{-4}\right)$. The $b \bar{b}$ mode constrains a NP model only in the heavy mass range of $m_{b \bar{b}} \geq 800 \mathrm{GeV}$ [63] due to the huge QCD background, where both $\mathcal{B}(H \rightarrow b \bar{b})$ and $\mathcal{B}(A \rightarrow b \bar{b})$ are extremely small because of the dominant decays into $b^{\prime} \bar{b}^{\prime}$.

\section{A. Constraints from direct searches on $A$}

A meaningful constraint is from the neutral Higgs boson searches at the LEP through $e^{+} e^{-} \rightarrow H_{i} H_{j}\left(H_{i, j}=h, H\right.$, $A$ ) in the framework of the minimal supersymmetric standard model [64]. The analysis was based on four different decay channels of $4 b, 2 b 2 \tau$, and $4 \tau$. Since the searches span center-of-mass energies from $91 \mathrm{GeV}$ to $209 \mathrm{GeV}$, the heavy $C P$-even $H$ cannot be produced kinematically. The $C P$-odd scalar boson $A$ is produced in association with $h$, mediated by the $Z$ boson. With the observed Higgs boson mass of $125 \mathrm{GeV}$, the LEP result excludes $M_{A} \lesssim 65 \mathrm{GeV}$. This is consistent with the exclusion from $\mathcal{B}_{\mathrm{BSM}}^{h}$, as discussed below Eq. (24).

We now consider the resonance searches in the $\tau \tau$ channel at the LHC. Both ATLAS and CMS experiments presented their results based on the run-1 $[65,66]$ and run-2 $[67,68]$ data. Since the LHC run- 1 data at $\sqrt{s}=7+8 \mathrm{TeV}$ had weaker constraints, we focus on the run-2 results. In Fig. 8, we show $\sigma \times \mathcal{B}(A \rightarrow \tau \tau)$ in the 2HDM-SM4 at the 13-TeV LHC. As shown in the next subsection, the pseudoscalar $A$ gets a much stronger constraint from the $\tau \tau$ channel than the $C P$-even $H$. This is partly because the gluon fusion production of $A$ is more efficient due to a larger loop function than that of $H$ (see Fig. 7). Another reason for this is the larger branching ratio of $A \rightarrow \tau \tau$ because of the absence of $A \rightarrow W W / Z Z / h h$. Both ATLAS and CMS experiments exclude the parameter space with $t_{\beta} \geq 4$ and $M_{A} \leq 2 M_{b^{\prime}}$. When $t_{\beta}=3$ (the minimum value of $t_{\beta}$ allowed by the observed $\kappa_{V}$ ), two experiments yield different lower bounds on $M_{A}$. Upon the absence of a combined ATLAS and CMS analysis, we take a conservative stance on constraining the model, i.e., adopting the weaker constraint between two experiment results. For $t_{\beta}=3, M_{A} \lesssim 350 \mathrm{GeV}$ is excluded at the $95 \%$ C.L.

The smoking-gun signature for $A$ at the LHC is the $Z h$ channel, followed by $Z \rightarrow \ell \ell$ and $h \rightarrow b \bar{b} / \tau \tau$ [74-76]. In the SM, $p p \rightarrow Z h$ proceeds mainly through $q \bar{q} \rightarrow Z h$, mediated by $Z^{*}$. The gluon fusion production also occurs in the SM through the quark triangle diagram and the quark box diagram, Figs. 9(a) and 9(c). In the SM, $\sigma(g g \rightarrow Z h)$ is small, about $10 \%$ of the $q \bar{q}$ annihilation process, mainly because of the destructive interference between the triangle and box diagrams [77]. In the 2HDM-SM4, there are four kinds of new contributions: (i) $\sigma(q \bar{q} \rightarrow Z h)$ is reduced by a factor of $\kappa_{V}^{2}$; (ii) $\mathcal{B}(h \rightarrow b \bar{b} / \tau \tau)$ in the exact wrong-sign

TABLE I. Summary of the direct searches that are efficient for $H / A$ in the 2HDM-SM4 at high energy colliders.

\begin{tabular}{lccc}
\hline \hline Process & Target & Mass range & Experiment \\
\hline$e^{+} e^{-} \rightarrow 4 b, 4 \tau, b \bar{b} \tau \tau$ & $A$ & {$\left[2 m_{\tau}, 100 \mathrm{GeV}\right]$} & LEP [64] \\
$p p \rightarrow \tau \tau$ & $H, A$ & {$[100 \mathrm{GeV}, 1 \mathrm{TeV}]$} & LHC run 1 [65,66] \\
$p p \rightarrow Z Z^{(*)}$ & $H$ & {$[90 \mathrm{GeV}, 3.2 \mathrm{TeV}]$} & LHC run 2 [67,68] \\
$p p \rightarrow Z h$ & $A$ & {$[110 \mathrm{GeV}, 1 \mathrm{TeV}]$} & LHC run 2 [69-72] \\
\hline \hline
\end{tabular}




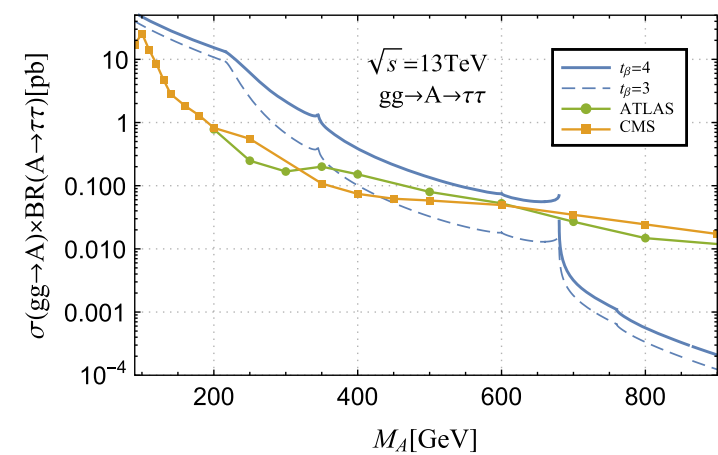

FIG. 8. We show $\sigma(g g \rightarrow A) \times \mathcal{B}(A \rightarrow \tau \tau)$ as a function of $M_{A}$ at the $13-\mathrm{TeV}$ LHC for $t_{\beta}=3$ and $t_{\beta}=4$. We apply the NNLO $K$ factor to the heavy Higgs resonance production by using the HIGLU package [57]. For comparison, we also show the 95\% C.L. upper limits on $\sigma \times \mathcal{B}(\phi \rightarrow \tau \tau)$ data $[67,68]$.

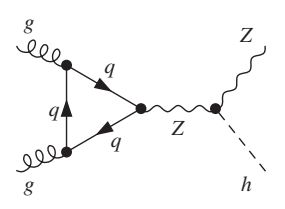

(a)

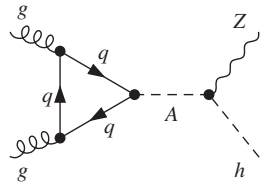

(b)

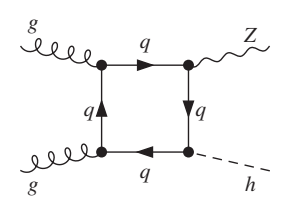

(c)
FIG. 9. Feynman diagrams for the $g g \rightarrow Z h$ process in the 2HDM-SM4. Here $q$ denotes all of the four generation quarks, including $t^{\prime}$ and $b^{\prime}$.

limit is increased because of smaller $\Gamma(h \rightarrow W W / Z Z)$ than in the SM but the same $\Gamma(h \rightarrow b \bar{b} / \tau \tau)$; (iii) the fourth generation quarks contribute to all of the loop diagrams for $g g \rightarrow Z h$; (iv) new triangle diagrams mediated by $A$ appear. In what follows, we call Fig. 9(a) the Z-triangle diagram, Fig. 9(b) the A-triangle diagram, and Fig. 9(c) the box diagram.

In order to see the interference effects in detail, we split the scattering amplitude into the $Z$-triangle part $\left(\mathscr{M}_{Z}\right)$, the $A$-triangle part $\left(\mathscr{M}_{A}\right)$, and the box part $\left(\mathscr{M}_{\square}\right)$. The partonlevel cross section becomes

$$
\begin{aligned}
\hat{\sigma}= & \frac{1}{32 \pi \hat{s}} \int d \cos \theta^{*} \bar{\sum}\left[\left|\mathscr{M}_{Z}\right|^{2}+\left|\mathscr{M}_{A}\right|^{2}+\left|\mathscr{M}_{\square}\right|^{2}\right. \\
& \left.+2 \Re e\left(\mathscr{M}_{Z} \mathscr{M}_{A}^{*}\right)+2 \mathfrak{R} e\left(\mathscr{M}_{Z} \mathscr{M}_{\square}^{*}\right)+2 \mathfrak{R} e\left(\mathscr{M}_{A} \mathscr{M}_{\square}^{*}\right)\right] \\
\equiv & \hat{\sigma}_{Z}+\hat{\sigma}_{A}+\hat{\sigma}_{\square}+\hat{\sigma}_{Z A}+\hat{\sigma}_{Z \square}+\hat{\sigma}_{A \square},
\end{aligned}
$$

where $\theta^{*}$ is the scattering angle in the center-of-mass frame and $\bar{\sum}$ denotes the proper summation and average over helicities and colors. Figure 10 shows the individual contributions to the $m_{Z h}$ distribution of the full $g g \rightarrow Z h$ for $t_{\beta}=4$ and $M_{A}=800 \mathrm{GeV}$ with $\Gamma_{\text {tot }}^{A}=2.0 \mathrm{TeV}$ at the $13-\mathrm{TeV}$ LHC. We use $K_{q \bar{q}}=1.31$ and $K_{g g}=2.1$ to match up with the updated Higgs calculation results [78,79], and we assume the same $K$ factor for the NP calculation. The cross section only from the $Z$-triangle diagrams, $d \sigma_{Z}$,

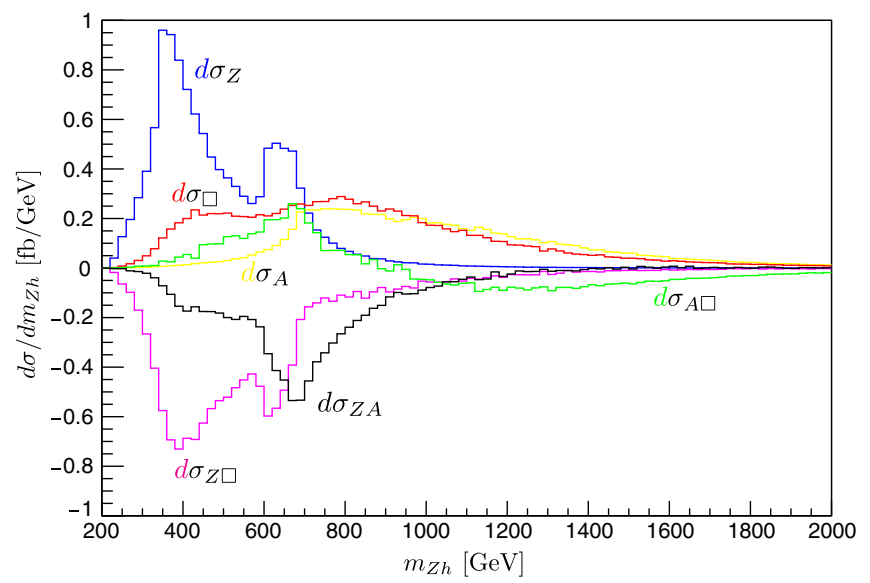

FIG. 10. Invariant mass $m_{Z h}$ distribution for $g g \rightarrow Z h$ production for $t_{\beta}=4$ and $M_{A}=800 \mathrm{GeV}$ at $\sqrt{s}=13 \mathrm{TeV}$. We plot individual contributions from the $Z$-triangle, $A$-triangle, box diagrams, and their interference terms. We use $K_{q \bar{q}}=1.31$ and $K_{g g}=2.1[78,79]$.

shows threshold behaviors around $m_{Z h} \simeq 2 m_{t}, \quad 2 M_{t^{\prime}}$, $2 M_{b^{\prime}}$. The contribution from $t^{\prime}$ and $b^{\prime}$ is rather small because the corresponding transition amplitude is proportional to the axial vector coupling of the $Z$ boson, $g_{A}^{Z f f}=$ $-\left(T_{3}^{f}\right)_{L} / 2$ [79]. For almost degenerate masses of $t^{\prime}$ and $b^{\prime}$, two contributions are canceled. On the other hand, $d \sigma_{\square}$ has a large signal rate in the heavy $m_{Z h}$ range because the opposite sign between $g_{A}^{Z b^{\prime} b^{\prime}}$ and $g_{A}^{Z t^{\prime} t^{\prime}}$ is compensated by the opposite sign between $Y_{b^{\prime}}^{h}$ and $Y_{t^{\prime}}^{h}$. The $d \sigma_{A} / d m_{Z h}$ shows a very wide resonance shape, resulting in a small signal rate. Both $d \sigma_{Z \square}$ and $d \sigma_{Z A}$ yield destructive interference in the whole $m_{Z h}$ region, large enough to almost cancel $d \sigma_{Z}$. The interference between the $A$-triangle and box diagrams is constructive for $m_{Z h} \leq M_{A}$, while it is destructive for $m_{Z h} \geq M_{A}$, a typical peak-dip structure [80]. In summary, the contributions from the interference are as large as noninterference ones.

In Fig. 11, we present the total invariant mass distribution in the SM and two 2HDM-SM4 cases: one with $t_{\beta}=4$ and $M_{A}=800 \mathrm{GeV}$ (pink line) and another with $t_{\beta}=3$ and $M_{A}=500 \mathrm{GeV}$ (blue line). We use $K_{q \bar{q}}=1.31$ and $K_{g g}=2.1[78,79]$. For both NP cases, we calculate the full Feynman diagrams in Fig. 9. The total $m_{Z h}$ distribution for $t_{\beta}=4$ and $M_{A}=800 \mathrm{GeV}$, of which the individual contributions are shown in Fig. 10, shows a peculiar shape with two thresholds of $t \bar{t}$ and $b^{\prime} \bar{b}^{\prime}$ followed by a very slow downhill slope. This bizarre distribution is the consequence of large interference effects. The case with $t_{\beta}=3$ and $M_{H}=500 \mathrm{GeV}$ with $\Gamma_{\text {tot }}^{A}=13.8 \mathrm{GeV}$ yields a prominent peak over the SM main background of $q \bar{q} \rightarrow Z h$. The LHC experiments cannot miss the peak. Indeed, the total cross section of $p p \rightarrow Z h$ for $t_{\beta}=3$ and $M_{A}=$ $500 \mathrm{GeV}$ is about $24.8 \mathrm{pb}$, far above the upper bound 


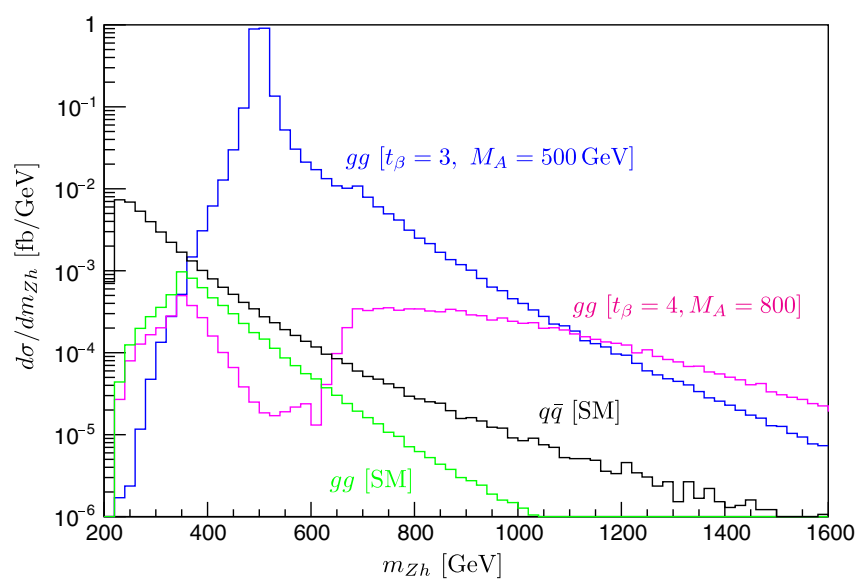

FIG. 11. Invariant mass $m_{Z h}$ distribution for the $p p \rightarrow Z h$ process at $\sqrt{s}=13 \mathrm{TeV}$. We separately present the $q \bar{q}$ contributions and the $g g$ contributions in the SM and 2HDM-SM4. We consider two NP cases: (i) $t_{\beta}=3$ and $M_{A}=500 \mathrm{GeV}$ and (ii) $t_{\beta}=4$ and $M_{A}=800 \mathrm{GeV}$ in the benchmark scenario in Eq. (25). We use $K_{q \bar{q}}=1.31$ and $K_{g g}=2.1[78,79]$.

on $\sigma \times \mathcal{B}(A \rightarrow Z h) \simeq 0.854 \mathrm{pb}$ at $M_{A}=500 \mathrm{GeV}$. Larger $t_{\beta}$ does not help to allow the model when $M_{A} \lesssim 2 M_{b^{\prime}}$ : (i) The resonance peak becomes more prominent because of a smaller width for larger $t_{\beta}$ in this mass range, as shown in Fig. 6; (ii) $t_{\beta} \gtrsim 4$ for $M_{A}<2 M_{b^{\prime}}$ is already excluded by the $\tau \tau$ resonance searches as in Fig. 8. When $M_{A} \gtrsim 2 M_{b^{\prime}}$, the width becomes too wide to show a resonance peak. We calculate the partially integrated cross section for the excess in each $m_{Z h}$ bin, defined in Eq. (28), and compare with the ATLAS result of the upper bounds on $\sigma \times \mathcal{B}(A \rightarrow Z h)$ [75]. We find that $t_{\beta}=4$ and $M_{A}=800 \mathrm{GeV}$ are still allowed. This conclusion is valid for larger $t_{\beta}$ and $M_{A}>2 M_{b^{\prime}}$.

\section{B. Constraints from direct searches for $\boldsymbol{H}$}

For $C P$-even $H$, we first study the constraint from the resonance searches in the $\tau \tau$ channel based on the ATLAS and CMS run-2 data [67,68]. Figure 12 shows $\sigma \times \mathcal{B}(H \rightarrow$ $\tau \tau)$ in the 2HDM-SM4 at the 13-TeV LHC. In the benchmark scenario, we consider two cases of $t_{\beta}=3$ and $t_{\beta}=4$. For the $K$ factor, we take the NNLO result from the HIGLU package [57]. Compared with the rapid drop of $\mathcal{B}(H \rightarrow \tau \tau)$ as a function of $M_{H}$ in Fig. $4, \sigma \times \mathcal{B}(H \rightarrow \tau \tau)$ decreases slowly. This is because of the sizable cross section of the gluon fusion production of $H$ for heavy $M_{H}$ (see Fig. 7). The current LHC data in the $\tau \tau$ final states meaningfully exclude $M_{H}$, though less than the pseudoscalar $A$. Adopting the weaker constraint between two experiment results, $M_{H}<180 \mathrm{GeV}\left(M_{H}<350 \mathrm{GeV}\right)$ is excluded for $t_{\beta}=$ $3\left(t_{\beta}=4\right)$ at the $95 \%$ C.L.

The smoking-gun signature for the $C P$-even $H$ is from the $Z Z$ final state $[69,70]$. In the SM, the production of a $Z$ boson pair is mainly through the $q \bar{q}$ annihilation. The gluon

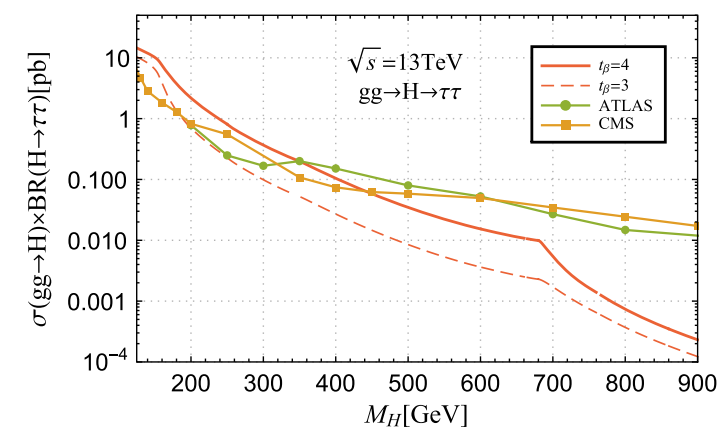

FIG. 12. We show $\sigma(g g \rightarrow H) \times \mathcal{B}(H \rightarrow \tau \tau)$ as a function of $M_{H}$ at the 13-TeV LHC. For two cases of $t_{\beta}=3$ and $t_{\beta}=4$, we set $M_{t^{\prime}}=300 \mathrm{GeV}, M_{b^{\prime}}=340 \mathrm{GeV}, M_{\nu^{\prime}}=430 \mathrm{GeV}$, and $M_{\tau^{\prime}}=380 \mathrm{GeV}$. We apply the NNLO $K$ factor to the heavy Higgs resonance production by using the HIGLU package [57]. We also show the ATLAS and CMS 95\% C.L. upper bounds on $\sigma \times \mathcal{B}(\phi \rightarrow \tau \tau)$.

fusion production via the quark loops is subleading, of which the cross section is about $10 \%$ of the Drell-Yan process. In the 2HDM-SM4, the Drell-Yan process $q \bar{q} \rightarrow$ $\mathrm{ZZ}$ is not affected since the CKM mixing $V_{4 i}$ is extremely suppressed. The gluon fusion process has three kinds of Feynman diagrams, as shown in Fig. 13: (a) the triangle diagrams mediated by $h$, (b) the triangle diagrams mediated by $H$, and (c) the box diagrams. New contributions are from the fourth generation quarks running in the loops and from the $H$-triangle diagram.

Figure 14 shows the noninterference contributions to $d \sigma / d m_{Z Z}(g g \rightarrow Z Z)$ from the $h$-triangle, $H$-triangle, and box diagrams as a function of $m_{Z Z}$ at the 13-TeV LHC. We set $t_{\beta}=4$ and $M_{H}=900 \mathrm{GeV}$ and include $K_{g g}=1.8$ and $K_{q \bar{q}}=1.5$. The definition of $d \sigma_{i}$ is given in Eq. (30). The box diagrams $\left(d \sigma_{\square}\right)$ yield the continuum background with a monotonically decreasing slope against $m_{Z Z}$, but the fourth generation quarks in the loop decrease the slope around the $b^{\prime} \bar{b}^{\prime}$ threshold. In terms of the total signal rate, the contribution from the box diagrams is dominant. The $H$-triangle diagrams yield a very wide resonance peak around $M_{A}=900 \mathrm{GeV}$. In the range of $m_{Z Z} \gtrsim 1 \mathrm{TeV}$, the contribution from the $H$-triangle diagrams is as large as that from the box diagrams. On the other hand, the $h$-triangle diagrams make a negligible contribution in the whole range of $m_{Z Z}$. The total distribution denoted by the black line

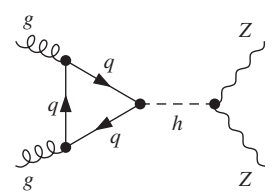

(a)

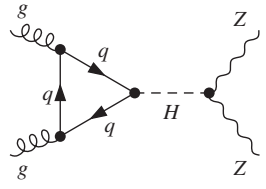

(b)

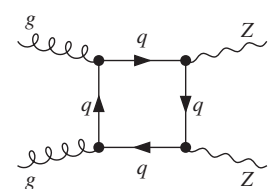

(c)
FIG. 13. Feynman diagrams for the $g g \rightarrow Z Z$ process in the 2HDM-SM4. Here $q$ denotes all of the four generation quarks, including $t^{\prime}$ and $b^{\prime}$. 


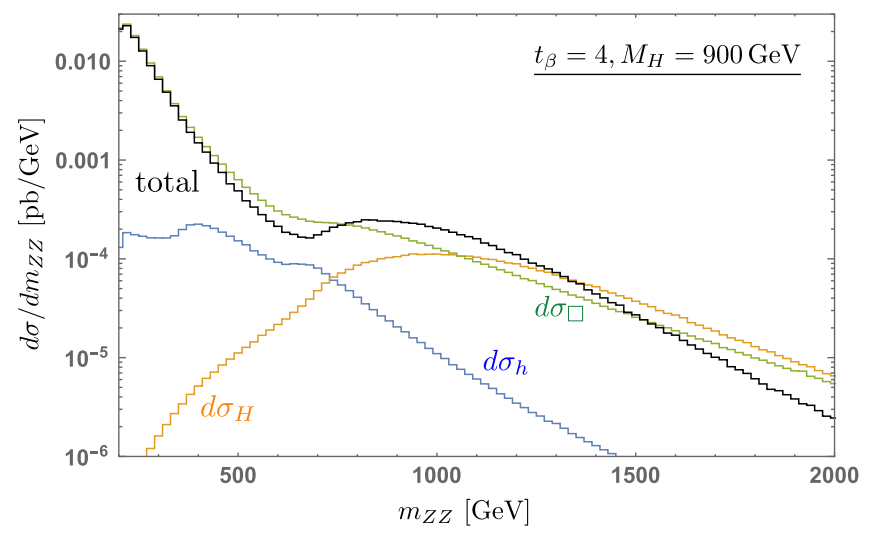

FIG. 14. The differential cross section for $g g \rightarrow Z Z$ against $m_{Z Z}$ in the 2HDM-SM4 at the 13-TeV LHC. We set $t_{\beta}=4$ and $M_{H}=900 \mathrm{GeV}$. We show individual contributions from the $h$ triangle, $H$-triangle, and box diagrams. We include $K_{g g}=1.8$ [81] and $K_{q \bar{q}}=1.5$ [82].

shows some deviation from the simple sum of $d \sigma_{\square}+d \sigma_{H}+d \sigma_{h}$, especially around $m_{Z Z} \sim 700 \mathrm{GeV}$. Obviously, the interference plays a role.

In Fig. 15, we show the three kinds of interference effects, $d \sigma_{i j}$ defined in Eq. (30), that are too small to show, together with noninterference $d \sigma_{i}$ in a single plot. First, the interference between the $h$-triangle and box diagrams, $\sigma_{h \square}$, is destructive and largest. We observe the threshold effects around $2 m_{t}$ and $2 M_{b^{\prime}}$. The interference $d \sigma_{H \square}$ shows a very asymmetric and wide peak-dip structure [83], practically like a wide peak of constructive interference. The opposite sign between $d \sigma_{h \square}$ and $d \sigma_{H \square}$ is attributed to the opposite sign between the $h b^{\prime} \bar{b}^{\prime}$ and $H b^{\prime} \bar{b}^{\prime}$ couplings. Finally, $d \sigma_{h H}$ is destructive but negligible.

Figure 16 shows the total invariant mass $m_{Z Z}$ distributions of $q \bar{q} \rightarrow Z Z$ in the SM, and $g g \rightarrow Z Z$ in the SM and the 2HDM-SM4. We consider three NP cases: (i) $t_{\beta}=3$ and $M_{H}=500 \mathrm{GeV}$, (ii) $t_{\beta}=4$ and $M_{H}=900 \mathrm{GeV}$, and (iii) $t_{\beta}=7$ and $M_{H}=900 \mathrm{GeV}$. It is clear to see that the

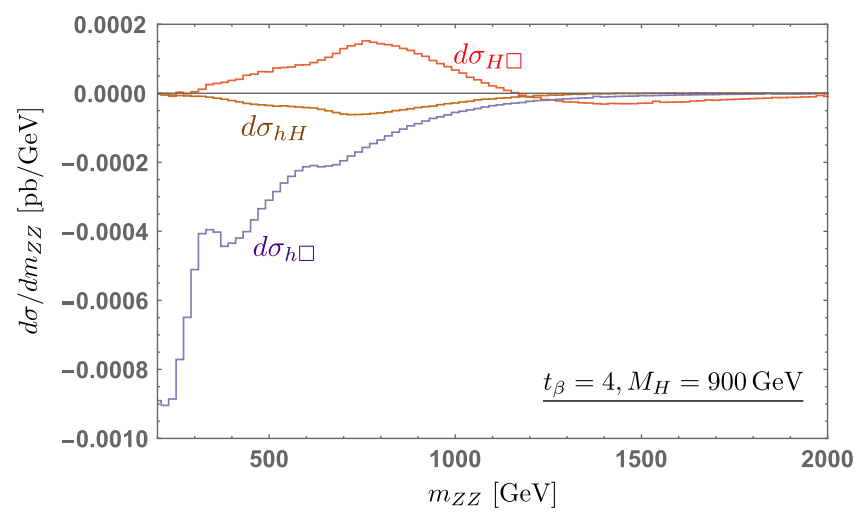

FIG. 15. The differential cross section for $g g \rightarrow Z Z$ against $m_{Z Z}$ from the interference terms. We set $t_{\beta}=4$ and $M_{H}=900 \mathrm{GeV}$ at the 13-TeV LHC. We include $K_{g g}=1.8$ [81] and $K_{q \bar{q}}=1.5$ [82].

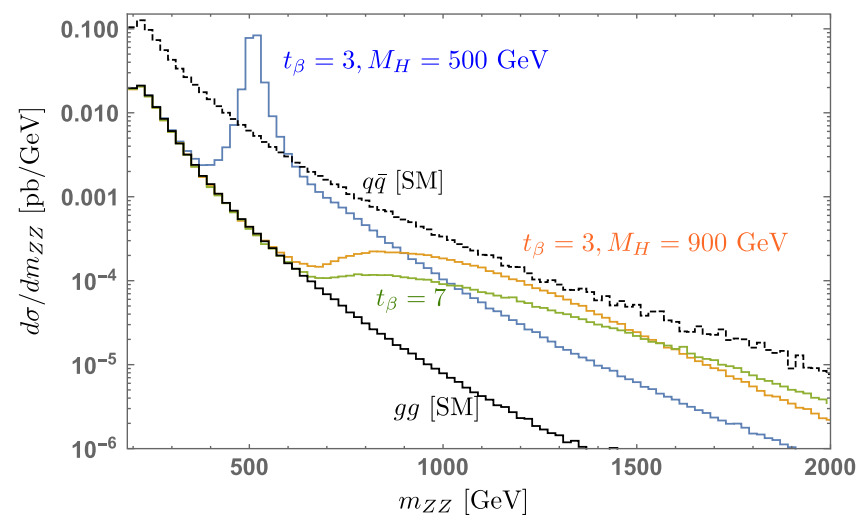

FIG. 16. The differential cross section against $m_{Z Z}$ in the SM and 2HDM-SM4 at the 13-TeV LHC. We consider three NP cases: $t_{\beta}=3$ and $M_{H}=500 \mathrm{GeV} ; t_{\beta}=4$ and $M_{H}=900 \mathrm{GeV}$; $t_{\beta}=7$ and $M_{H}=900 \mathrm{GeV}$ for the benchmark point. We include $K_{g g}=1.8$ [81] and $K_{q \bar{q}}=1.5$ [82].

case of $t_{\beta}=3$ and $M_{H}=500 \mathrm{GeV}$, which was allowed by the $\tau \tau$ constraint in Fig. 12, yields a very outstanding peak because of a relatively narrow width and large production rate of $g g \rightarrow H$. Apparently, the current upper bound on $\sigma \times \mathcal{B}(\phi \rightarrow Z Z)$ excludes this case. Note that the whole parameter space with $M_{H}<2 M_{b^{\prime}}$ (where $H$ remains as a prominent peak) is excluded since larger $t_{\beta}$ yields a larger production cross section of $g g \rightarrow H$ as shown in Fig. 7 .

When $M_{H}$ is above the $b^{\prime} \bar{b}^{\prime}$ threshold, as shown by two cases of $t_{\beta}=4,7$ with $M_{H}=900 \mathrm{GeV}$, the very large width of $H$ spreads the $H$ resonance peak. It can be seen that the signal rate of $g g \rightarrow Z Z$ in the 2HDM-SM4 is compatible with the SM Drell-Yan production rate in the mass range of $m_{Z Z} \gtrsim 1 \mathrm{TeV}$. Comparing the $t_{\beta}=4$ $\left(\Gamma_{\text {tot }}^{H}=1.3 \mathrm{TeV}\right)$ and $t_{\beta}=7\left(\Gamma_{\text {tot }}^{H}=3.6 \mathrm{TeV}\right)$ cases, we find that the dependence on $t_{\beta}$ is not dramatically different once $M_{H}$ is beyond the $b^{\prime} \bar{b}^{\prime}$ threshold. In order to probe this very wide resonance, we adopt the method suggested at the end of Sec. VA, utilizing the 95\% C.L. upper limits on $\sigma \times \mathcal{B}(H \rightarrow Z Z)$ for each invariant mass bin. Since the CMS Collaboration presented the results for $\Gamma_{X}=100 \mathrm{GeV}$, we take the CMS results [69].

In Fig. 17, we show the partially integrated cross section from NP effects only, $\Delta \sigma^{\mathrm{NP}}$, for various $m_{Z Z}$ bins. The sizes of the bins are based on the CMS results [69]. We set $M_{H}=$ $900 \mathrm{GeV}$ and consider two cases of $t_{\beta}=4$ and $t_{\beta}=7$. The red region is excluded by the $95 \%$ C.L. upper bounds on $\sigma \times \mathcal{B}(H \rightarrow Z Z)$. Both $t_{\beta}=4$ and $t_{\beta}=7$ cases are excluded by the bin of $m_{Z Z} \in[1,1.5] \mathrm{TeV}$. Larger $t_{\beta}$, apart from the breakdown of the perturbativity of $Y_{b^{\prime}}^{H}$, does not help us to decrease the signal rate of NP since the gluon fusion production cross section increases with larger $t_{\beta}$. Note that we cannot increase $M_{H}$ substantially further since $M_{H}=900 \mathrm{GeV}$ is almost a maximally allowed value by the theoretical constraint (see Fig. 1). In summary, the 


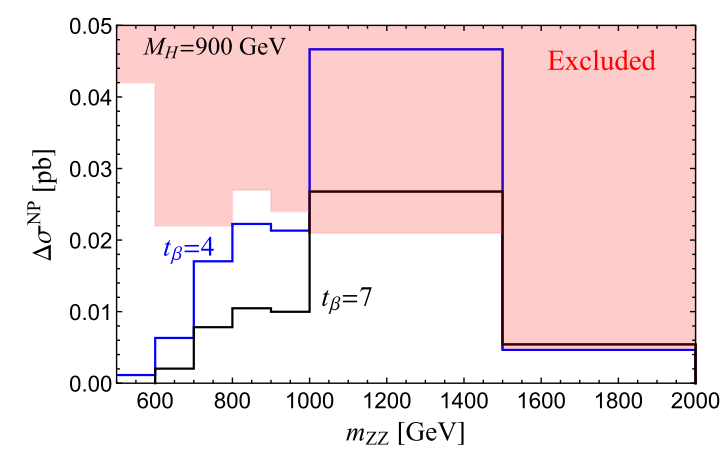

FIG. 17. Partially integrated cross section of NP effects only, $\Delta \sigma^{\mathrm{NP}}$ defined in Eq. (28), for various $m_{Z Z}$ bins. We set $M_{H}=$ $900 \mathrm{GeV}$ and consider $t_{\beta}=4$ and $t_{\beta}=7$. The red region is excluded by the $95 \%$ C.L. upper bounds on $\sigma \times \mathcal{B}(H \rightarrow Z Z)$.

current LHC data on the $Z Z$ channel, along with theoretical constraints, exclude the 2HDM-SM4 at leading order.

\section{CONCLUSIONS}

We have studied the theoretical and phenomenological constraints on the type-II two-Higgs-doublet model with a sequential fourth fermion generation in the exact wrongsign limit, the 2HDM-SM4. The SM fermion sector is extended to accommodate an additional chiral fermion generation of which the masses are generated by the same Higgs mechanism. Upon the absence of new fermion signals at high energy colliders, the fourth generation fermion $F$ should be heavy, which greatly enhances the $h F F$ couplings. The loop-induced Higgs coupling modifiers such as $\kappa_{g}, \kappa_{\gamma}$, and $\kappa_{Z \gamma}$ become SM-like if the up-type and down-type fermions have opposite Higgs coupling modifiers: $\kappa_{\text {up }}=1$ and $\kappa_{\text {down }}=-1$. We call this the exact wrongsign limit, which can be realized in the type-II 2HDM.

We have studied the following constraints: (i) the theoretical constraints on the scalar potential from the boundedfrom-below potential, unitarity, perturbativity, and the vacuum stability; (ii) the LHC and Tevatron search bounds on the fourth generation fermion masses; (iii) the $\bar{B} \rightarrow X_{s} \gamma$ bound on $M_{H^{ \pm}}$; (iv) the electroweak oblique parameters $\Delta S$ and $\Delta T$; (v) the observed Higgs coupling modifiers, including the exotic Higgs decay rate; (vi) the LEP searches for $e^{+} e^{-} \rightarrow A h$; and (vii) the LHC scalar resonance searches in the $\tau \tau, Z h$, and $Z Z$ modes. Based on this comprehensive and thorough study, we come to the conclusion that the 2HDM-SM4 is excluded at leading order. Two features of the 2HDM-SM4 play a crucial role here: the exact wrong-sign limit and the very large Yukawa couplings of the down-type fourth generation fermions with $H$ and $A, Y_{b^{\prime}, \tau^{\prime}}^{H / A}=\tan \beta M_{F} / v$.

The exact wrong-sign limit requiring $\beta+\alpha=\pi / 2$ constrains the model very differently than the alignment limit of $\beta-\alpha=\pi / 2$. First, the theoretical constraints do not allow the decoupling limit, which puts upper bounds like
$M_{H}, M_{A} \lesssim 920 \mathrm{GeV}$ and $M_{H^{ \pm}} \lesssim 620 \mathrm{GeV}$. Second, the constraint on $\tan \beta$ from the Higgs precision data works in the opposite way compared to that from the unitarity of $Y_{b^{\prime}, \tau^{\prime}}^{H / A}=\tan \beta M_{F} / v$ : The observed $\kappa_{V}\left(=s_{\beta-\alpha}\right)$ at $95 \%$ C.L. requires $\tan \beta \gtrsim 3$, while the unitarity of $Y_{b^{\prime}, \tau^{\prime}}^{H / A}$ requires $\tan \beta \lesssim 8$. The allowed value of $\tan \beta \in[3,8]$ is not large enough to achieve the alignment limit: $\kappa_{V}\left(=s_{\beta-\alpha}\right)$ deviates significantly from 1 and thus $c_{\beta-\alpha}$ has a sizable value. Therefore, the usual 2HDM safety zone in the alignment and decoupling limit cannot be reached in the 2HDM-SM4.

These indirect constraints set the basic characteristics of the decays of $H$ and $A$. Two features are to be noted. First, the sizable $c_{\beta-\alpha}$ leads to significant decays of $H \rightarrow$ $W W / Z Z / h h$ and $A \rightarrow Z h$, which would have been absent in the alignment limit. Above the $W W / Z Z(Z h)$ and below the $b^{\prime} \bar{b}^{\prime}$ threshold, $H(A)$ decays dominantly into $W W / Z Z / h h(Z h)$. After the $b^{\prime} \bar{b}^{\prime}$ threshold, the enhanced Yukawa coupling $Y_{b^{\prime}}^{H / A}$ makes the decay of $H / A \rightarrow b^{\prime} \bar{b}^{\prime}$ dominant. Because of the sizable $\Gamma(H / A \rightarrow g g)$ and nonnegligible $\mathcal{B}(H / A \rightarrow \tau \tau)$, the LHC direct searches in the $\tau \tau$ channel impose the strong constraint: For $t_{\beta}=4, M_{A}<$ $2 M_{b^{\prime}}$ and $M_{H}<350 \mathrm{GeV}$ are excluded; for $t_{\beta}=3, M_{A} \lesssim$ $350 \mathrm{GeV}$ and $M_{H}<180 \mathrm{GeV}$ are excluded at the $95 \%$ C.L.

The smoking-gun signatures of $A$ and $H$ are the LHC direct searches in the $Z h$ and $Z Z$ channels, respectively. Since their total decay widths are not small enough to adopt the narrow-width approximation, we performed the full calculation including the SM continuum background without resorting to $\sigma \times \mathcal{B}$. The interference effects turned out to be very important. In the $g g \rightarrow Z h$ process, they are as much as the noninterference ones. We found that both $A$ and $H$ with the mass below the $b^{\prime} \bar{b}^{\prime}$ threshold are excluded by the current LHC data at the $95 \%$ C.L. Above the $b^{\prime} \bar{b}^{\prime}$ threshold, $\Gamma_{\text {tot }}^{H / A}$ becomes very large and is compatible with the mass $M_{H / A}$. The invariant mass distributions spread very widely. Beyond relying on the total event rates, we suggested a method to utilize the upper bounds on $\sigma \times \mathcal{B}$ on each invariant mass bin, through the partially integrated cross section. Although the pseudoscalar $A$ with $M_{A}>$ $2 M_{b^{\prime}}$ is allowed by the current LHC data in the $Z h$ channel, the $C P$-even heavy scalar $H$ is excluded by the current LHC data in the $Z Z$ channel.

We conclude that the 2HDM-SM4 is excluded by the combination of the theoretical and experimental constraints at leading order. Before finishing, however, we would like to point out two issues. First, we assumed nearly degenerate heavy scalar masses as well as degenerate fourth generation fermion masses for the oblique $\Delta T$ parameter. Sizable mass differences are still possible if the contribution from heavy scalars to $\Delta T$ is canceled by that from the fourth generation fermions. However, we found that the upper bound on $M_{H}$, which played a crucial role in excluding the model, is not changed much by relaxing the degenerate condition: The 
conclusion remains the same. A more crucial limitation of our conclusion is that our results are based on leading-order calculations. The conclusion may be relaxed if we consider the next-to-leading order corrections. The sizable $\cos (\beta-\alpha)$, the multiplying factor for the $H V V$ and $A Z h$ couplings at tree level, plays a decisive role in excluding the model. With the fourth generation fermions running in the loop, both $H V V$ and $A Z h$ couplings can be meaningfully different from the tree-level results. The constraints from the LHC resonance searches in the $Z Z$ and $Z h$ final states may be alleviated.

\section{ACKNOWLEDGMENTS}

The work of S. K. K. was supported by NRF Grant No. 2017K1A3A7A09016430. The work of Z. Q. was supported by IBS under the project code IBS-R018-D1. The work of J.S. was supported by the National Research Foundation of Korea, Grant No. NRF2016R1D1A1B03932102. Y. W. Y. was supported by the Basic Science Research Program through the National Research Foundation of Korea (NRF) funded by the Ministry of Education (2017R1A6A3A11036365).
[1] P. H. Frampton, P. Q. Hung, and M. Sher, Phys. Rep. 330, 263 (2000).

[2] D. Choudhury, T. M. P. Tait, and C. E. M. Wagner, Phys. Rev. D 65, 053002 (2002).

[3] M. Bobrowski, A. Lenz, J. Riedl, and J. Rohrwild, Phys. Rev. D 79, 113006 (2009).

[4] G. Aad et al. (ATLAS Collaboration), Phys. Lett. B 716, 1 (2012).

[5] S. Chatrchyan et al. (CMS Collaboration), Phys. Lett. B 716, 30 (2012).

[6] D. Das, A. Kundu, and I. Saha, Phys. Rev. D 97, 011701 (2018).

[7] G. Bhattacharyya, D. Das, and P. B. Pal, Phys. Rev. D 87, 011702 (2013).

[8] D. Carmi, A. Falkowski, E. Kuflik, and T. Volansky, J. High Energy Phys. 07 (2012) 136.

[9] C. W. Chiang and K. Yagyu, J. High Energy Phys. 07 (2013) 160.

[10] P. M. Ferreira, J. F. Gunion, H. E. Haber, and R. Santos, Phys. Rev. D 89, 115003 (2014).

[11] D. Fontes, J. C. Romo, and J. P. Silva, Phys. Rev. D 90, 015021 (2014).

[12] N. Chen and H. J. He, J. High Energy Phys. 04 (2012) 062.

[13] A. I. Hernndez-Jurez, A. Moyotl, and G. Tavares-Velasco, Phys. Rev. D 98, 035040 (2018).

[14] L. Wang, R. Shi, and X. F. Han, Phys. Rev. D 96, 115025 (2017).

[15] S. Chamorro-Solano, A. Moyotl, and M. A. Prez, J. Phys. G 45, 075003 (2018).

[16] G. C. Branco, P. M. Ferreira, L. Lavoura, M. N. Rebelo, M. Sher, and J. P. Silva, Phys. Rep. 516, 1 (2012).

[17] S. L. Adler, Phys. Rev. 177, 2426 (1969).

[18] J. S. Bell and R. Jackiw, Nuovo Cimento A 60, 47 (1969).

[19] S. L. Glashow and S. Weinberg, Phys. Rev. D 15, 1958 (1977).

[20] E. A. Paschos, Phys. Rev. D 15, 1966 (1977).

[21] M. D. Campos, D. Cogollo, M. Lindner, T. Melo, F. S. Queiroz, and W. Rodejohann, J. High Energy Phys. 08 (2017) 092.

[22] S. Chang, S. K. Kang, J. P. Lee, and J. Song, Phys. Rev. D 92, 075023 (2015).
[23] J. Bernon, J. F. Gunion, H. E. Haber, Y. Jiang, and S. Kraml, Phys. Rev. D 93, 035027 (2016).

[24] J. F. Gunion and H. E. Haber, Phys. Rev. D 67, 075019 (2003).

[25] A. Djouadi, Phys. Rep. 457, 1 (2008).

[26] P. M. Ferreira, R. Guedes, M. O. P. Sampaio, and R. Santos, J. High Energy Phys. 12 (2014) 067.

[27] I. F. Ginzburg and M. Krawczyk, Phys. Rev. D 72, 115013 (2005).

[28] A. Dighe, D. Ghosh, R. M. Godbole, and A. Prasath, Phys. Rev. D 85, 114035 (2012).

[29] S. Dawson and P. Jaiswal, Phys. Rev. D 82, 073017 (2010).

[30] S. Chatrchyan et al. (CMS Collaboration), Phys. Rev. D 86, 112003 (2012).

[31] A. Lister (CDF Collaboration), arXiv:0810.3349.

[32] T. Aaltonen et al. (CDF Collaboration), Phys. Rev. Lett. 104, 091801 (2010).

[33] C. J. Flacco, D. Whiteson, T. M. P. Tait, and S. Bar-Shalom, Phys. Rev. Lett. 105, 111801 (2010).

[34] C. J. Flacco, D. Whiteson, and M. Kelly, Phys. Rev. D 83, 114048 (2011).

[35] C. Patrignani et al. (Particle Data Group), Chin. Phys. C 40, 100001 (2016).

[36] M. Misiak and M. Steinhauser, Eur. Phys. J. C 77, 201 (2017).

[37] M. Misiak, Acta Phys. Pol. B 48, 2173 (2017).

[38] A. Abdesselam et al. (Belle Collaboration), arXiv: 1608.02344.

[39] P. M. Ferreira, R. Guedes, J. F. Gunion, H. E. Haber, M. O. P. Sampaio, and R. Santos, arXiv:1407.4396.

[40] I. P. Ivanov, Phys. Rev. D 75, 035001 (2007); 76, 039902(E) (2007).

[41] B. W. Lee, C. Quigg, and H. B. Thacker, Phys. Rev. D 16, 1519 (1977).

[42] S. Kanemura and K. Yagyu, Phys. Lett. B 751, 289 (2015).

[43] A. Arhrib, arXiv:hep-ph/0012353.

[44] A. Barroso, P. M. Ferreira, I. P. Ivanov, and R. Santos, J. High Energy Phys. 06 (2013) 045.

[45] G. D. Kribs, T. Plehn, M. Spannowsky, and T. M. P. Tait, Phys. Rev. D 76, 075016 (2007). 
[46] W. Grimus, L. Lavoura, O. M. Ogreid, and P. Osland, J. Phys. G 35, 075001 (2008).

[47] W. Grimus, L. Lavoura, O. M. Ogreid, and P. Osland, Nucl. Phys. B801, 81 (2008).

[48] G. Aad et al. (ATLAS and CMS Collaborations), J. High Energy Phys. 08 (2016) 045.

[49] M. Aaboud et al. (ATLAS Collaboration), J. High Energy Phys. 03 (2018) 095.

[50] A. M. Sirunyan et al. (CMS Collaboration), J. High Energy Phys. 11 (2017) 047.

[51] Y. W. Yoon, K. Cheung, S. K. Kang, and J. Song, Phys. Rev. D 96, 055041 (2017).

[52] M. Aaboud et al. (ATLAS Collaboration), Phys. Rev. D 98, 052005 (2018).

[53] CMS Collaboration, CERN, Report No. CMS-PAS-HIG16-040, 2017.

[54] S. Chatrchyan et al. (CMS Collaboration), Phys. Rev. D 89, 092007 (2014).

[55] M. Aaboud et al. (ATLAS Collaboration), Phys. Rev. D 96, 052004 (2017).

[56] R. Franceschini, G. F. Giudice, J. F. Kamenik, M. McCullough, A. Pomarol, R. Rattazzi, M. Redi, F. Riva, A. Strumia, and R. Torre, J. High Energy Phys. 03 (2016) 144.

[57] M. Spira, arXiv:hep-ph/9510347.

[58] A. Djouadi, Phys. Rep. 459, 1 (2008).

[59] D. J. Miller, S. Moretti, D. P. Roy, and W. J. Stirling, Phys. Rev. D 61, 055011 (2000).

[60] G. Aad et al. (ATLAS Collaboration), Phys. Rev. Lett. 113, 171801 (2014).

[61] CMS Collaboration, CERN, Report No. CMS-PAS-HIG17-013, 2017.

[62] G. Aad et al. (ATLAS Collaboration), Phys. Lett. B 759, 601 (2016).

[63] M. Aaboud et al. (ATLAS Collaboration), Phys. Rev. D 98, 032016 (2018).

[64] S. Schael et al. (ALEPH, DELPHI, L3, and OPAL Collaborations, LEP Working Group for Higgs Boson Searches), Eur. Phys. J. C 47, 547 (2006).

[65] G. Aad et al. (ATLAS Collaboration), J. High Energy Phys. 11 (2014) 056.
[66] V. Khachatryan et al. (CMS Collaboration), J. High Energy Phys. 10 (2014) 160.

[67] M. Aaboud et al. (ATLAS Collaboration), J. High Energy Phys. 01 (2018) 055.

[68] A. M. Sirunyan et al. (CMS Collaboration), J. High Energy Phys. 09 (2018) 007.

[69] A. M. Sirunyan et al. (CMS Collaboration), J. High Energy Phys. 06 (2018) 127.

[70] M. Aaboud et al. (ATLAS Collaboration), J. High Energy Phys. 03 (2018) 009.

[71] V. Khachatryan et al. (CMS Collaboration), J. High Energy Phys. 10 (2015) 144.

[72] G. Aad et al. (ATLAS Collaboration), Eur. Phys. J. C 76, 45 (2016).

[73] V. Khachatryan et al. (CMS Collaboration), Phys. Lett. B 748, 221 (2015).

[74] G. Aad et al. (ATLAS Collaboration), Phys. Lett. B 744, 163 (2015).

[75] ATLAS Collaboration, CERN, Report No. ATLAS-CONF2016-015, 2016.

[76] CMS Collaboration, CERN, Report No. CMS-PAS-HIG18-005, 2018.

[77] C. Englert, M. McCullough, and M. Spannowsky, Phys. Rev. D 89, 013013 (2014).

[78] D. de Florian et al. (LHC Higgs Cross Section Working Group), Handbook of LHC Higgs Cross Sections: 4. Deciphering the Nature of the Higgs Sector, CERN Yellow Reports: Monographs, Vol. 2 (CERN, Geneva, Switzerland, 2017).

[79] L. M. Carpenter, T. Han, K. Hendricks, Z. Qian, and N. Zhou, Phys. Rev. D 95, 053003 (2017).

[80] S. Jung, Y. W. Yoon, and J. Song, Phys. Rev. D 93, 055035 (2016).

[81] F. Caola, K. Melnikov, R. Röntsch, and L. Tancredi, Phys. Rev. D 92, 094028 (2015).

[82] F. Cascioli, T. Gehrmann, M. Grazzini, S. Kallweit, P. Maierhöfer, A. von Manteuffel, S. Pozzorini, D. Rathlev, L. Tancredi, and E. Weihs, Phys. Lett. B 735, 311 (2014).

[83] S. Jung, J. Song, and Y. W. Yoon, Phys. Rev. D 92, 055009 (2015). 